\title{
EFFECT OF ELECTRIC FIELD ON DISPERSION OF A SOLUTE IN AN MHD FLOW THROUGH A VERTICAL CHANNEL WITH AND WITHOUT CHEMICAL REACTION
}

\author{
J.C. UMAVATHI and J.P. KUMAR \\ Department of Mathematics, Gulbarga University \\ Gulbarga, Karnataka, INDIA 585106 \\ E-mails: drumavathi@rediffmail.com; p_rathap@yahoo.com \\ R.S.R. GORLA* and B.J. GIREESHA \\ Department of Mechanical Engineering \\ Cleveland State University \\ Cleveland-44115, OHIO, USA \\ Department of Studies and Research in Mathematics \\ Kuvempu University \\ Shankaraghatta-577 451, Shimoga, Karnataka, INDIA \\ E-mail: r.gorla@csuohio.edu; g.bijjanaljayanna@csuohio.edu
}

\begin{abstract}
The longitudinal dispersion of a solute between two parallel plates filled with two immiscible electrically conducting fluids is analyzed using Taylor's model. The fluids in both the regions are incompressible and the transport properties are assumed to be constant. The channel walls are assumed to be electrically insulating. Separate solutions are matched at the interface using suitable matching conditions. The flow is accompanied by an irreversible first-order chemical reaction. The effects of the viscosity ratio, pressure gradient and Hartman number on the effective Taylor dispersion coefficient and volumetric flow rate for an open and short circuit are drawn in the absence and in the presence of chemical reactions. As the Hartman number increases the effective Taylor diffusion coefficient decreases for both open and short circuits. When the magnetic field remains constant, the numerical results show that for homogeneous and heterogeneous reactions, the effective Taylor diffusion coefficient decreases with an increase in the reaction rate constant for both open and short circuits.
\end{abstract}

Key words: Taylor dispersion, immiscible fluids, conducting fluid, MHD, chemical reaction.

\section{Introduction}

A wide application of the dispersion model began fifty five years ago when numerous authors noticed that longitudinal mixing can be treated the same as diffusion; see Levenspiel and Smith [1]. The most notable is the work of Danckwerts [2] and Taylor [3-5] who in his pioneering papers introduced the concept of longitudinal dispersion superimposed on plug flow to describe the fact that not all fluid elements travel at equal speed through a system.

Taylor [3-5] investigated the way in which a liquid spreads out longitudinally as it moves down a straight tube and demonstrated by a few careful experiments and a novel mathematical analysis of a rather heuristic kind, that for downstream of the source the longitudinal spread is equivalent to a diffusion process; he also provided estimates for the longitudinal dispersion coefficient. Since then the notion of a longitudinal dispersion has been recognized as being relevant in a wide variety of contexts, like in flows in rivers and estuaries, in oil pipelines, in water mains, in pneumatic and hydraulic industrial devices, in blood vessels, in tubules, in paints. An enormous variety of extensions and generalizations of Taylor's simple result for a

\footnotetext{
" To whom correspondence should be addressed
} 
steady flow in a straight circular tube has been developed; see Batchelor [6]. The most notable developments of the classical asymptotic theory of Taylor, while still preserving the basic ideas of his original work, were produced by Aris [7], Horn [8], and Brenner [9, 10]. Considerable attention has also been paid to the onedimensional dispersion of the solute during relatively short times, too short for the macro transport process to be fully established; see Philip [11], Gill and Sankarasubramanian [12, 13], DeGance and Johns [14-15], Hatton and Lightfoot [16-18], Yamanaka [19-21], and Smith [22, 23]. The importance for practice of the diffusion analysis of Taylor and the subsequent investigations lies in the ability of the one-dimensional transport equation to take into account complicated velocity and concentration profiles in the same manner, as well as providing a theoretical framework for the dispersion coefficient.

But in the above papers, it was assumed that there is no chemical reaction of any kind between the solute and the fluid during the course of dispersion. This was not always true. In many physical phenomena, homogeneous and heterogeneous chemical reactions are always present. Such an analysis of a finite first-order homogeneous reaction in a laminar pipe flow was first studied both theoretically and experimentally by Cleland and Wilhem [24]. The effect of the heterogeneous reaction taking place at the wall was discussed by Katz [25], whereas Walker [26], Soloman and Hudson [27], investigated the combined effect of first-order heterogeneous and homogeneous chemical reactions on the dispersion of soluble matter in a parallel plate channel flow.

The flow aspects of immiscible fluids are of special importance. In modeling such problems, the presence of a second immiscible fluid phase adds a number of complexities as to the nature of interacting transport phenomena and interface conditions between the phases. In general, multi-phase flows are driven by gravitational and viscous forces. There has been some theoretical and experimental work on a stratified laminar flow of two immiscible fluids in a horizontal pipe (Packham and Shail [28], Alireza and Sahai [29], Malashetty and Leela [30, 31]). Loharsbi and Sahai [32] studied two-phase MHD flow and heat transfer in a parallel plate channel with one of the fluids being electrically conducting. A two-phase MHD flow and heat transfer in an inclined channel was investigated by Malashetty and Umavathi [33]. Chamkha [34] reported analytical solutions for the flow of two-immiscible fluids in porous and non-porous parallel-plate channels. Later on, a magnetohydrodynamic two-fluid convective flow and heat transfer in a composite porous medium was analyzed by Malashetty et al. [35-37]. Umavathi et al. [38] analyzed the Poiseuille-Couette flow of two immiscible fluids between two inclined parallel plate. Recently, Umavathi and Shekar [39] studied the fully developed laminar mixed convection flow in a vertical wavy channel filled with two immiscible viscous fluids with traveling thermal waves using the perturbation method. Kumar et al. [40, 41] studied the solute dispersion in a composite porous medium

Keeping in view the wide area of practical importance of multi-fluid flows as mentioned above, it is the objective of the present study to investigate the dispersion of the solute in an electrically conducting immiscible channel flow.

\section{Mathematical formulation of the problem}

The physical configuration considered in this study is shown in Fig.1. Consider the laminar flow of two immiscible fluids between two parallel plates distant $2 h$ apart, taking the $X$-axis along the mid-section of the channel and the $Y$-axis perpendicular to the walls. The magnetic field $B_{0}$ is applied normal to the flow field and the electric field is applied along the flow field. Region-1 $(-h \leq Y \leq 0)$ is filled with the electrically conducting fluid of conductivity $\sigma_{e l}$, density $\rho_{l}$, viscosity $\mu_{l}$, under a uniform pressure gradient $\frac{d P_{1}}{d X}$, whereas region-2 $(0 \leq Y \leq h)$ is filled with another electrically conducting fluid of conductivity $\sigma_{e 2}$, density $\rho_{2}$, viscosity $\mu_{2}$, under a uniform pressure gradient $\frac{d P_{2}}{d X}$. The fluids in both the regions are Newtonian fluids. 


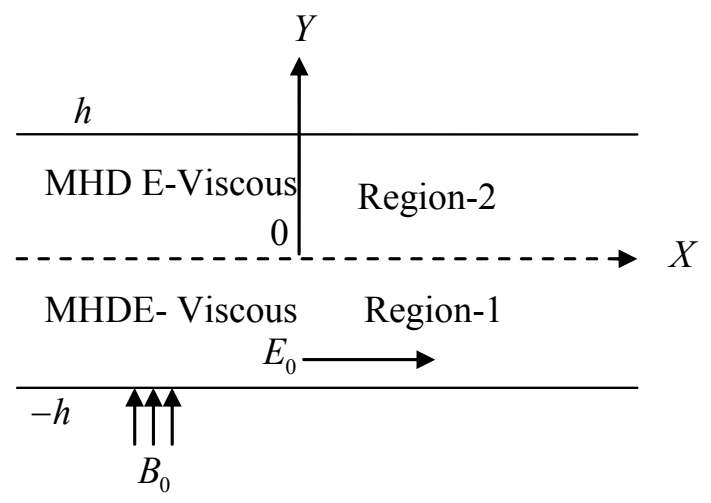

Fig.1. Physical configuration.

It is assumed that the flow is steady, laminar, fully developed, and that fluid properties are constant. The flow in both the regions is assumed to be driven by a common constant pressure gradient. Under these assumptions, the governing equations of motion for incompressible fluids are Region-1

$$
\mu_{1} \frac{d^{2} U_{1}}{d Y^{2}}-\frac{d P_{1}}{d X}-\sigma_{e l} B_{0}^{2} U_{1}-\sigma_{e l} B_{0} E_{0}=0
$$

Region-2

$$
\mu_{2} \frac{d^{2} U_{2}}{d Y^{2}}-\frac{d P_{2}}{d X}-\sigma_{e 2} B_{0}^{2} U_{2}-\sigma_{e 2} B_{0} E_{0}=0
$$

where $U_{i}$ is the $X$-component of fluid velocity and $P_{i}$ is the pressure. The subscripts 1 and 2 denote the values for region- 1 and region-2, respectively.

The boundary conditions on velocity are no-slip conditions requiring that the velocity must vanish at the walls. In addition, continuity of velocity and shear stress at the interface is assumed. With these assumptions, the boundary and interface conditions on velocity become

$$
\begin{aligned}
& U_{1}=0 \text { at } Y=-h, \\
& U_{2}=0 \text { at } Y=h, \\
& U_{1}=U_{2} \text { at } Y=0, \\
& \mu_{1} \frac{d U_{1}}{d Y}=\mu_{2} \frac{d U_{2}}{d Y} \text { at } Y=0 .
\end{aligned}
$$

Using the non-dimensional parameters

$$
\begin{aligned}
& \eta=\frac{Y}{h}, \quad u_{1}=\frac{\rho_{1} h}{\mu_{1}} U_{1}, \quad u_{2}=\frac{\rho_{2} h}{\mu_{2}} U_{2}, \quad x=\frac{X}{h}, \quad p_{1}^{*}=\frac{P_{1}}{\rho_{l}\left(v_{l} / h\right)^{2}}, \\
& p_{2}^{*}=\frac{P_{2}}{\rho_{2}\left(v_{2} / h\right)^{2}}, \quad M=B_{0} h \sqrt{\frac{\sigma_{e l}}{\mu_{l}}}, \quad E=\frac{\rho_{1} h E_{0}}{\mu_{1} B_{0}},
\end{aligned}
$$

in Eqs (2.1) to (2.3) become 
Region-1

$$
\frac{d^{2} u_{1}}{d \eta^{2}}-\frac{d p_{1}^{*}}{d x}-M^{2} u_{1}-M^{2} E=0
$$

Region-2

$$
\begin{aligned}
& \frac{d^{2} u_{2}}{d \eta^{2}}-\frac{d p_{2}^{*}}{d x}-\frac{\sigma_{r} M^{2}}{m} u_{2}-\frac{\sigma_{r} M^{2} E}{m^{2} n}=0, \\
& u_{1}=0 \quad \text { at } \quad \eta=-1, \\
& u_{2}=0 \quad \text { at } \quad \eta=1, \\
& u_{1}=m n u_{2}, \quad \frac{d u_{1}}{d \eta}=m^{2} n \frac{d u_{2}}{d \eta} \text { at } \eta=0
\end{aligned}
$$

where

$$
m=\mu_{2} / \mu_{1} \quad \text { and } \quad n=\rho_{1} / \rho_{2}, \quad \sigma_{r}=\frac{\sigma_{e 2}}{\sigma_{e 1}} .
$$

Solutions of Eqs (2.5) and (2.6) are

$$
\begin{aligned}
& u_{1}=a_{1} \cosh (M \eta)+a_{2} \sinh (M \eta)-\frac{A}{M^{2}}, \\
& u_{2}=a_{3} \cosh (B \eta)+a_{4} \sinh (B \eta)-\frac{c}{B^{2}} .
\end{aligned}
$$

From Eqs (2.8) and (2.9) the average velocities become

$$
\begin{aligned}
& \bar{u}_{1}=\frac{1}{2} \int_{-1}^{0} u_{1} d \eta, \\
& \bar{u}_{2}=\frac{1}{2} \int_{0}^{1} u_{2} d \eta .
\end{aligned}
$$

\section{Case 1: Diffusion of a tracer in the absence of a first-order chemical reaction.}

The equation for the concentration $C_{1}$ of the solute for the region- 1 satisfies

$$
\frac{\partial C_{1}}{\partial t}+u_{1} \frac{\partial C_{1}}{\partial X}=D_{1}\left(\frac{\partial^{2} C_{1}}{\partial X^{2}}+\frac{\partial^{2} C_{1}}{\partial Y^{2}}\right)
$$

Similarly, the equation for the concentration $C_{2}$ of the solute for the region-2 satisfies

$$
\frac{\partial C_{2}}{\partial t}+u_{2} \frac{\partial C_{2}}{\partial X}=D_{2}\left(\frac{\partial^{2} C_{2}}{\partial X^{2}}+\frac{\partial^{2} C_{2}}{\partial Y^{2}}\right)
$$

in which $D_{1}$ and $D_{2}$ are the molecular diffusion coefficients (assumed constants) for the region-1 and region-2, respectively. 
If we now consider convection across a plane moving with the mean speed of the flow, then relative to this plane the fluid velocities are given by

Region-1

Region-2

$$
u_{1 x}=u_{1}-\bar{u}=a_{1} \cosh (M \eta)+a_{2} \sinh (M \eta)+l_{1} .
$$

$$
u_{2 x}=u_{2}-\bar{u}=a_{3} \cosh (B \eta)+a_{4} \sinh (B \eta)+l_{2}
$$

where $\bar{u}$ is the sum of average velocities of region-1 and region- 2 .

Introducing the dimensionless quantities

$$
\theta_{1}=\frac{t_{1}}{\bar{t}_{1}}, \quad \bar{t}_{1}=\frac{L_{1}}{\bar{u}_{1}}, \quad \xi_{1}=\frac{x_{1}-\bar{u}_{1} t}{L}, \quad \theta_{2}=\frac{t_{2}}{\bar{t}_{2}}, \quad \bar{t}_{2}=\frac{L_{2}}{\bar{u}_{2}}, \quad \xi_{2}=\frac{x_{2}-\bar{u}_{2} t}{L},
$$

and using Eqs (2.14) and (2.15), Eqs (2.12) and (2.13) (assuming that $\frac{\partial^{2} C_{1}}{\partial X^{2}}<\frac{\partial^{2} C_{1}}{\partial Y^{2}}$ and $\frac{\partial^{2} C_{2}}{\partial X^{2}}<<\frac{\partial^{2} C_{2}}{\partial Y^{2}}$ ) become Region-1

Region-2

$$
\frac{1}{t} \frac{\partial C_{1}}{\partial \theta_{1}}+\frac{u_{1 x}}{L} \frac{\partial C_{1}}{\partial \xi_{1}}=\frac{D_{1}}{h^{2}} \frac{\partial^{2} C_{1}}{\partial \eta^{2}}
$$

$$
\frac{1}{t} \frac{\partial C_{2}}{\partial \theta_{2}}+\frac{u_{2 x}}{L} \frac{\partial C_{2}}{\partial \xi_{2}}=\frac{D_{2}}{h^{2}} \frac{\partial^{2} C_{2}}{\partial \eta^{2}}
$$

where $L$ is the typical length along the flow direction. Following Taylor [3], we now assume that partial equilibrium is established in any cross-section of the channel so that the variations of $C_{1}$ and $C_{2}$ with $\eta$ are calculated from Eqs (2.17) and (2.18) as

Region-1

$$
\frac{\partial^{2} C_{1}}{\partial \eta^{2}}=\frac{h^{2}}{D_{1} L} u_{1 x} \frac{\partial C_{1}}{\partial \xi_{1}} .
$$

Region-2

$$
\frac{\partial^{2} C_{2}}{\partial \eta^{2}}=\frac{h^{2}}{D_{2} L} u_{2 x} \frac{\partial C_{2}}{\partial \xi_{2}}
$$

To solve these equations we use the following boundary conditions

$$
\frac{\partial C_{1}}{\partial \eta}=0 \quad \text { at } \quad \eta=-1 \quad \text { and } \quad \frac{\partial C_{2}}{\partial \eta}=0 \text { at } \eta=1 .
$$

Equations (2.19) and (2.20) are solved exactly for $C_{1}$ and $C_{2}$ which are given by Region-1

$$
C_{1}=Z_{1}\left(\frac{a_{1}}{M^{2}} \cosh (M \eta)+\frac{a_{2}}{M^{2}} \sinh (M \eta)+\frac{l_{1}}{2} \eta^{2}\right)+b_{1} \eta+b_{2}
$$

Region-2

$$
C_{2}=Z_{2}\left(\frac{a_{3}}{B^{2}} \cosh (B \eta)+\frac{a_{4}}{B^{2}} \sinh (B \eta)+\frac{l_{2}}{2} \eta^{2}\right)+b_{3} \eta+b_{4} .
$$

where $b_{2}$ and $b_{4}$ are constants to be determined using the entry conditions. 
The volumetric flow rates at which the solute is transported across a section of the channel of unit breadth $Q_{1}$ (region-1) and $Q_{2}$ (region-2) using Eqs (2.16), (2.17) and (2.22), (2.23), respectively are, given by

$$
\begin{aligned}
& Q_{1}=h \int_{-1}^{0} C_{1} u_{1 x} d \eta=-Z_{1} h \int_{-1}^{0} C_{11} u_{1 x} d \eta \\
& Q_{2}=h \int_{0}^{1} C_{2} u_{2 x} d \eta=-Z_{2} h \int_{0}^{1} C_{22} u_{2 x} d \eta \\
& C_{11}=-\left(\frac{a_{1} \cosh (M \eta)}{M^{2}}+\frac{a_{2} \sinh (M \eta)}{M^{2}}+\frac{l_{1} \eta^{2}}{2}+b_{11} \eta\right), \\
& C_{22}=-\left(\frac{a_{3} \cosh (B \eta)}{B^{2}}+\frac{a_{4} \sinh (B \eta)}{B^{2}}+\frac{l_{2} \eta^{2}}{2}+b_{32} \eta\right) .
\end{aligned}
$$

where

Following Taylor [3], we assume that the variations of $C_{1}$ and $C_{2}$ with $\eta$ are small compared with those in the longitudinal direction, and if $C_{m 1}$ and $C_{m 2}$ are the mean concentration over a section, $\partial C_{1} / \partial \xi_{1}$ and $\partial C_{2} / \partial \xi_{2}$ are indistinguishable from $\partial C_{m 1} / \partial \xi_{1}$ and $\partial C_{m 2} / \partial \xi_{2}$, respectively, so that Eqs (2.24) and (2.25) may be written as

Region-1

$$
Q_{1}=-D_{1}^{*} \frac{\partial C_{m 1}}{\partial \xi_{1}}
$$

Region-2

$$
Q_{2}=-D_{2}^{*} \frac{\partial C_{m 2}}{\partial \xi_{2}}
$$

The fact that no material is lost in the process is expressed by the continuity equation for $C_{m l}$ and $C_{m 2}$, namely:

Region-1

$$
\frac{\partial Q_{1}}{\partial \xi_{1}}=-2 \frac{\partial C_{m 1}}{\partial t}
$$

Region-2

$$
\frac{\partial Q_{2}}{\partial \xi_{2}}=-2 \frac{\partial C_{m 2}}{\partial t}
$$

Region-1

Equations (2.28) and (2.29) using Eqs (2.24) and (2.25) become

Region-2

$$
\frac{\partial C_{m 1}}{\partial t}=\frac{D_{1}^{*}}{2} \frac{\partial^{2} C_{m 1}}{\partial \xi_{1}^{2}} .
$$

$$
\frac{\partial C_{m 2}}{\partial t}=\frac{D_{2}^{*}}{2} \frac{\partial^{2} C_{m 2}}{\partial \xi_{2}^{2}},
$$

which are the equations governing the longitudinal dispersion, where 


$$
\begin{aligned}
& D_{1}^{*}=\frac{h^{2}}{2 D_{1}} \int_{-1}^{0} C_{11} u_{1 x} d \eta=\frac{h^{2}}{2 D_{1}} F_{1}\left(M, E, p_{1}, p_{2}, m, n\right), \\
& D_{2}^{*}=\frac{h^{2}}{2 D_{2}} \int_{-1}^{0} C_{12} u_{1 x} d \eta=\frac{h^{2}}{2 D_{2}} F_{2}\left(M, E, p_{1}, p_{2}, m, n\right) .
\end{aligned}
$$

Equations (2.30) and (2.31) are the well known heat conduction equations which can be solved easily for given initial conditions.

\section{Case 1b: Diffusion of a tracer in the absence of a first order chemical reaction and for purely viscous fluid (two fluid model)}

To validate the results of the present model, the problem is solved in the absence of the magnetic field and compared with the results of Gupta and Gupta [42].

The non-dimensional equations of motion for incompressible fluids are Region-1

$$
\frac{d^{2} u_{1}}{d y^{2}}-\frac{d p_{1}^{*}}{d x}=0
$$

Region-2

$$
\frac{d^{2} u_{2}}{d y^{2}}-\frac{d p_{2}^{*}}{d x}=0
$$

The boundary and interface conditions are defined as in Eq.(2.7). Using Eq.(2.7) in Eqs (32) and (33), the solutions become

$$
\begin{aligned}
& u_{1}=\frac{p_{1} \eta^{2}}{2}+a_{1} \eta+a_{2}, \\
& u_{2}=\frac{p_{2} \eta^{2}}{2}+a_{3} \eta+a_{4} .
\end{aligned}
$$

The average velocities become

$$
\begin{aligned}
& \bar{u}_{1}=\frac{1}{2}\left(\frac{p_{1}}{6}-\frac{a_{1}}{2}+a_{2}\right), \\
& \bar{u}_{2}=\frac{1}{2}\left(\frac{p_{1}}{6}+\frac{a_{3}}{2}+a_{4}\right) .
\end{aligned}
$$

The solutions of Eqs (2.19) and (2.20) in the absence of the applied magnetic field $B_{0}$ and applied electric field $E_{0}$ yields

$$
\begin{aligned}
& C_{1}=\frac{h^{2}}{D_{1} L} \frac{\partial C_{1}}{\partial \xi_{1}}\left(\frac{p_{1} \eta^{4}}{24}+\frac{a_{1} \eta^{3}}{6}+\frac{l c_{1} \eta^{2}}{2}+b_{1} \eta\right)+C_{01}, \\
& C_{2}=\frac{h^{2}}{D_{2} L} \frac{\partial C_{2}}{\partial \xi_{2}}\left(\frac{p_{2} \eta^{4}}{24}+\frac{a_{3} \eta^{3}}{6}+\frac{l c_{2} \eta^{2}}{2}+b_{3} \eta\right)+C_{02}
\end{aligned}
$$


where $C_{01}$ and $C_{02}$ being constants to be determined using entry conditions.

The volumetric rates at which the solute is transported across a section of the channel of unit breadth $Q_{1}$ (region-1) and $Q_{2}$ (region-2) and the effective dispersion coefficients $F_{i i}$ are evaluated as explained in the case 1a. The values of $F_{i}\left(p_{1}, p_{2}, m, n\right)$ are computed for different values of the dimensionless parameters $p_{i}$ and $m$ and are shown in Tab.4.

Case 1c: Diffusion of a tracer in the absence of a first order chemical reaction and for purely viscous fluid (one fluid model).

The non-dimensional equation of motion is

$$
\frac{d^{2} u}{d \eta^{2}}=\frac{d p}{d x}
$$

along with boundary conditions

$$
u=0 \quad \text { at } \quad \eta= \pm 1 .
$$

The solution of Eq.(2.40) is

$$
u=-p\left(1-\eta^{2}\right) / 2 \text {. }
$$

The average velocity is given by

$$
\bar{u}=-p / 3 .
$$

The concentration equation for one fluid model using Taylor [3] become

$$
\frac{\partial^{2} C}{\partial \eta^{2}}=\frac{h^{2}}{D L} \frac{\partial C}{\partial \xi} u_{x}
$$

where $\quad u_{x}=\frac{p \eta^{2}}{2}-\frac{p}{6}$.

The solution of Eq.(2.42) using boundary conditions $\frac{\partial C}{\partial \eta}=0$ at $\eta= \pm 1$ is

$$
C=\frac{h^{2}}{D L} \frac{\partial C}{\partial \xi}\left(\frac{p}{24} \eta^{4}-\frac{p}{12} \eta^{2}\right)+C_{0}
$$

where $C_{0}$ is a constant to be determined using entry conditions. breadth is

The volumetric flow rate in which the solute is transported across a section of the channel of unit

$$
Q=h \int_{-1}^{1} C u_{x} d \eta=-\frac{h^{2} p^{2}}{D} \frac{\partial C}{\partial \xi}\left(\frac{2}{945}\right)
$$

so that the value for $D^{*}$ can be written as $\frac{h^{2} p^{2}}{D} \frac{2}{945}$ by comparing with Fick's law of diffusion which agrees with the results of Wooding [43] where $p$ is the non-dimensional pressure gradient. $D^{*}$ is also the effective dispersion co-efficient obtained by Gupta and Gupta [42] in the absence of chemical reactions. 


\section{Case 2a: Diffusion of a tracer in the presence of a homogeneous first-order chemical reaction}

The physical model and the assumptions made in case 1 are true here, except that we have the chemical reaction. In this case we assume that the chemical reaction is first order and it occurs under such conditions that the gas film resistance is negligible. This means that the reaction term is $-K C_{1}$ (region-1) and $-K C_{2}$ (region-2) $\mathrm{mol} \mathrm{cm}^{-3} \mathrm{~s}^{-1}$, which represents the volume rate of disappearance of the solute due to the chemical reaction. Here $K$ represents the first-order reaction rate constant.

The velocity and average velocity are exactly the same as in Eqs (2.8)-(2.11). The equations for concentration, instead of Eqs (2.12) and (2.13), are

Region-1

$$
\frac{\partial C_{1}}{\partial t}+u_{1} \frac{\partial C_{1}}{\partial X}=D_{1}\left(\frac{\partial^{2} C_{1}}{\partial X^{2}}+\frac{\partial^{2} C_{1}}{\partial Y^{2}}\right)-K_{l} C_{1}
$$

Region-2

$$
\frac{\partial C_{2}}{\partial t}+u_{2} \frac{\partial C_{2}}{\partial X}=D_{2}\left(\frac{\partial^{2} C_{2}}{\partial X^{2}}+\frac{\partial^{2} C_{2}}{\partial Y^{2}}\right)-K_{2} C_{2}
$$

Along with the boundary condition (2.21), the continuity of concentration and continuity of mass flux at the interface is considered to evaluate the integrating constants. That is

$$
C_{1}=C_{2} \quad \text { and } \quad \frac{\partial C_{1}}{\partial \eta}=\frac{D_{2}}{D_{1}} \frac{\partial C_{2}}{\partial \eta} \text { at } \eta=0 .
$$
Region-1

Following the analysis of case 1, the non-dimensional form of Eqs (2.45) and (2.46) are

$$
\frac{\partial^{2} C_{1}}{\partial \eta^{2}}-\alpha_{1}^{2} C_{1}=\frac{h^{2}}{D_{1} L} u_{1 x} \frac{\partial C_{1}}{\partial \xi_{1}}
$$

Region-2

$$
\frac{\partial^{2} C_{2}}{\partial \eta^{2}}-\alpha_{2}^{2} C_{2}=\frac{h^{2}}{D_{2} L} u_{2 x} \frac{\partial C_{2}}{\partial \xi_{2}}
$$

where

$$
\alpha_{1}=h \sqrt{K_{1} / D_{1}} \quad \text { and } \quad \alpha_{2}=h \sqrt{K_{2} / D_{2}} \text {. }
$$

Region-1

The solutions of Eqs (2.48) and (2.49) become

$$
C_{l}=b_{1} \cosh \left(\alpha_{1} \eta\right)+b_{2} \sinh \left(\alpha_{1} \eta\right)+Z_{1}\left(\frac{a_{1} \cosh (M \eta)}{M^{2}-\alpha_{1}^{2}}+\frac{a_{2} \sinh (M \eta)}{M^{2}-\alpha_{1}^{2}}+\frac{l_{1}}{\alpha_{1}^{2}} \eta^{2}\right) .
$$

Region-2

$$
C_{2}=b_{3} \cosh \left(\alpha_{2} \eta\right)+b_{4} \sinh \left(\alpha_{2} \eta\right)+Z_{2}\left(\frac{a_{3} \cosh (B \eta)}{B^{2}-\alpha_{2}^{2}}+\frac{a_{4} \sinh (B \eta)}{B^{2}-\alpha_{2}^{2}}+\frac{l_{2}}{\alpha_{2}^{2}} \eta^{2}\right) .
$$

The expressions for $C_{1}$ and $C_{2}$ can also be written as

$$
C_{1}=\frac{h^{2}}{D_{1} L} \frac{\partial C_{1}}{\partial \xi_{1}} C_{11}+\frac{h^{2}}{D_{2} L} \frac{\partial C_{2}}{\partial \xi_{2}} C_{12}, \quad C_{2}=\frac{h^{2}}{D_{1} L} \frac{\partial C_{1}}{\partial \xi_{1}} C_{21}+\frac{h^{2}}{D_{2} L} \frac{\partial C_{2}}{\partial \xi_{2}} C_{22} .
$$


The volumetric flow rates at which the solute is transported across a section of the channel of unit breadth $Q_{1}$ (region-1) and $Q_{2}$ (region-2) using Eqs (2.14), (2.15) and (2.50), (2.51), respectively are given by Region-1

$$
Q_{1}=h \int_{-1}^{0} C_{1} u_{1 x} d \eta=-\left(Q_{11}+Q_{12}\right)
$$

Region-2

$$
Q_{2}=h \int_{0}^{l} C_{2} u_{2 x} d \eta=-\left(Q_{21}+Q_{22}\right)
$$

where

$$
\begin{array}{ll}
Q_{11}=-Z_{1} h \int_{-1}^{0} C_{11} u_{1 x} d \eta, & Q_{12}=-Z_{2} h \int_{-1}^{0} C_{12} u_{1 x} d \eta, \\
Q_{21}=-Z_{1} h \int_{0}^{1} C_{21} u_{2 x} d \eta, & Q_{22}=-Z_{2} h \int_{0}^{1} C_{22} u_{2 x} d \eta
\end{array}
$$

Following the procedure explained in case 1 and using the fact that no material is lost in the process expressed by the continuity equation for $C_{1}$ and $C_{2}$, given by Eqs (2.50) and (2.51), we obtain an effective dispersion coefficient $D^{*}$ in the form

$$
\begin{aligned}
& D_{11}^{*}=\frac{h^{2}}{2 D_{1}} \int_{-1}^{0} C_{11} u_{1 x} d \eta=\frac{h^{2}}{2 D_{1}} F_{11}\left(M, E, p_{1}, p_{2}, \alpha_{1}, \alpha_{2}, m, n\right), \\
& D_{12}^{*}=\frac{h^{2}}{2 D_{2}} \int_{-1}^{0} C_{12} u_{1 x} d \eta=\frac{h^{2}}{2 D_{2}} F_{12}\left(M, E, p_{1}, p_{2}, \alpha_{1}, \alpha_{2}, m, n\right), \\
& D_{21}^{*}=\frac{h^{2}}{2 D_{1}} \int_{-1}^{0} C_{21} u_{2 x} d \eta=\frac{h^{2}}{2 D_{1}} F_{21}\left(M, E, p_{1}, p_{2}, \alpha_{1}, \alpha_{2}, m, n\right), \\
& D_{22}^{*}=\frac{h^{2}}{2 D_{2}} \int_{-1}^{0} C_{22} u_{2 x} d \eta=\frac{h^{2}}{2 D_{2}} F_{22}\left(M, E, p_{1}, p_{2}, \alpha_{1}, \alpha_{2}, m, n\right) .
\end{aligned}
$$

Values of $F_{i i}$ are computed for different values of dimensionless parameters such as the Hartman number $\mathrm{M}$, viscosity ratio $m$ and pressure gradients $p_{1}, p_{2}$ for variations of $\alpha_{1}$ and $\alpha_{2}$ for both open and short circuits. The volumetric flow rate is also computed for variations of the Hartman number, viscosity ratio, pressure gradients and height of the channel.

\section{Case 2b: Diffusion of a tracer with a combined homogeneous and heterogeneous first-order chemical reaction}

We now discuss the problem of diffusion in a channel with a first-order irreversible chemical reaction taking place both in the bulk of the fluid as well as at the walls which are assumed to be catalytic. In this case the diffusion equations remain the same as defined in Eqs (2.48) and (2.49) subject to the dimensionless boundary and interface conditions as 


$$
\begin{aligned}
& \frac{\partial C_{1}}{\partial \eta}-\beta_{1} C_{1}=0 \text { at } \eta=-1, \\
& \frac{\partial C_{2}}{\partial \eta}+\beta_{2} C_{2}=0 \text { at } \eta=1, \\
& C_{1}=C_{2} \quad \text { at } \eta=0, \\
& D_{1} \frac{\partial C_{1}}{\partial \eta}=D_{2} \frac{\partial C_{2}}{\partial \eta} \text { at } \eta=0
\end{aligned}
$$

where $\beta_{1}=f_{1} h$ and $\beta_{2}=f_{2} h$ are the heterogeneous reaction rate parameters (or wall catalytic parameters) corresponding to the catalytic reaction at the walls.

The solutions of Eqs (2.48) and (2.49) are the same as in Eqs (2.50) and (2.51). The integrating constants $b_{1}, b_{2}, b_{3}$ and $b_{4}$ are obtained using boundary and interface conditions as defined in Eq.(2.55) and are given as follows

$$
b_{1}=Z_{1} b_{11}+Z_{2} b_{12}, \quad b_{2}=Z_{1} b_{21}+Z_{2} b_{22}, \quad b_{3}=Z_{1} b_{31}+Z_{2} b_{32}, \quad b_{4}=Z_{1} b_{41}+Z_{2} b_{42} .
$$

The procedure of evaluating the volumetric flow rate and effective dispersion coefficient is the same as in Eqs (2.52) to (2.54).

Case 2c: Diffusion of a tracer in the presence of a homogeneous first-order chemical reaction in the absence of the magnetic field for a purely viscous fluid (two fluid model).

We justify our results by comparing them with the results obtained by Gupta and Gupta [42] (one fluid model) with a first order chemical reaction for a purely viscous fluid.

The solutions of velocities and average velocities are the same as in Eqs (2.34) to (2.37). The solutions of Eqs (2.19) and (2.20) for a purely viscous fluid yields

$$
\begin{aligned}
& C_{1}=b_{1} \cosh \left(\alpha_{1} \eta\right)+b_{2} \sinh \left(\alpha_{1} \eta\right)+Z_{1}\left(l_{1} \eta^{2}+l_{2} \eta+l_{3}\right), \\
& C_{2}=b_{3} \cosh \left(\alpha_{2} \eta\right)+b_{4} \sinh \left(\alpha_{2} \eta\right)+Z_{2}\left(l_{4} \eta^{2}+l_{5} \eta+l_{6}\right) .
\end{aligned}
$$

The volumetric rates at which the solute is transported across a section of the channel of unit breadth $Q_{1}$ (region-1) and $Q_{2}$ (region-2) and the effective dispersion coefficients $F_{i i}$ are evaluated as explained in case1a. The values of $F_{i i}\left(\alpha_{1}, \alpha_{2}, p_{1}, p_{2}, m, n\right)$ are computed for different values of the dimensionless reaction rate parameters $\alpha_{i}, p_{i}$ and $m$ and are shown in Tab.4.

\section{Case 2d: The channel filled with only a viscous fluid (one fluid model) for a homogeneous chemical reaction.}

The solutions of velocities and average velocities are given in case 1c. The concentration equation for one fluid model using Taylor [3] becomes 


$$
\frac{\partial^{2} C}{\partial \eta^{2}}-\alpha^{2} C=\frac{h^{2}}{D L} \frac{\partial C}{\partial \xi} u_{x}
$$

where

$$
u_{x}=\frac{p \eta^{2}}{2}-\frac{p}{6}
$$

The solution of Eq.(2.58) using boundary conditions $\frac{\partial C}{\partial \eta}=0$ at $\eta= \pm 1$ is

$$
C=A \cosh (\alpha \eta)-\frac{h^{2}}{\alpha^{2} D L} \frac{\partial C}{\partial \xi}\left(\frac{p}{2} \eta^{2}-\frac{p}{6}+\frac{p}{\alpha^{2}}\right) .
$$

The volumetric flow rate in which the solute is transported across a section of the channel of unit breadth is

$$
Q=h \int_{-1}^{1} C u_{x} d \eta=\frac{h^{2} p^{2}}{\alpha^{2} D} \frac{\partial C}{\partial \xi}\left(\frac{1}{\alpha^{4}}+\frac{1}{3 \alpha^{2}}-\frac{\operatorname{coth}(\alpha)}{\alpha}-\frac{1}{45}\right)
$$

Comparing Eq.(2.60) with Fick's law of diffusion, we find that the solute is dispersed relative to a plane moving with the mean speed of the flow with an effective dispersion coefficient $D^{*}$ given by

$$
D^{*}=\frac{h^{2} p^{2}}{D} F(\alpha)
$$

where $\quad F(\alpha)=\frac{1}{\alpha^{2}}\left(\frac{\operatorname{coth}(\alpha)}{\alpha}-\frac{1}{\alpha^{4}}-\frac{1}{3 \alpha^{2}}+\frac{1}{45}\right)$.

Values of $F(\alpha)$ are computed for different values of the dimensionless reaction rate parameter $\alpha$ and are shown in Tab.4. When $\alpha \rightarrow 0$, Eq.(2.61) gives

$$
\lim _{\alpha \rightarrow 0} F(\alpha)=\frac{2}{945}
$$

so that the value for $D^{*}$ can be written as $\frac{h^{2} p^{2}}{D} \frac{2}{945}$ which agrees with the results of Wooding [43] where $p$ is the non-dimensional pressure gradient.

The solution for a heterogeneous chemical reaction is also found for two fluid and one fluid model and the results are shown in Tab.4. The constants which appeared in all the above equations are given in the Appendix.

\section{Results and discussion}

The longitudinal dispersion of a two-fluid MHD fluid flow between two parallel plates in the presence of a transverse magnetic field and uniform electric field applied across the channel is discussed. The dispersion of a solute is analyzed with or without a first order chemical reaction 
following the Taylor diffusion model. The average velocities in both the regions are evaluated using noslip conditions at the boundaries and continuity of velocity and shear stress at the interface. The effective Taylor diffusion coefficient (ETDC) in each region is evaluated for the governing parameters and is tabulated.

\section{Case 1: Diffusion of a tracer in the absence of a homogeneous first-order chemical reaction.}

The effect of Hartman number M on the velocity for open and short circuits is shown in Fig.1. We observe that the effect of increasing the magnetic field is to decrease the velocity in both the regions. We also observe the flattening of the velocity profile for large values of the Hartman number. This is due to the overall retarding effect of the Lorentz force $J \times B$. The Hartmann number represents the ratio of the Lorentz force to the viscous force. The velocity near the walls increases in order to keep the mass flow rate constant. This is the classical Hartmann result. Figure 1 also shows the effect of the electric field load parameter $E$ on the flow. The two cases $E=0$ (short circuit) and $E= \pm 1$ (open circuit) are considered in the graph. The effect of increasing $\mathrm{M}$ is quite opposite in the case when $E=-1$. In this case we observe that the electromagnetic force tends to accelerate the fluid. Again a positive $E$ accelerates the flow in opposite direction. Since we are considering the fluids in both the regions to be electrically conducting, we observe the symmetric profiles for both open and short circuits.

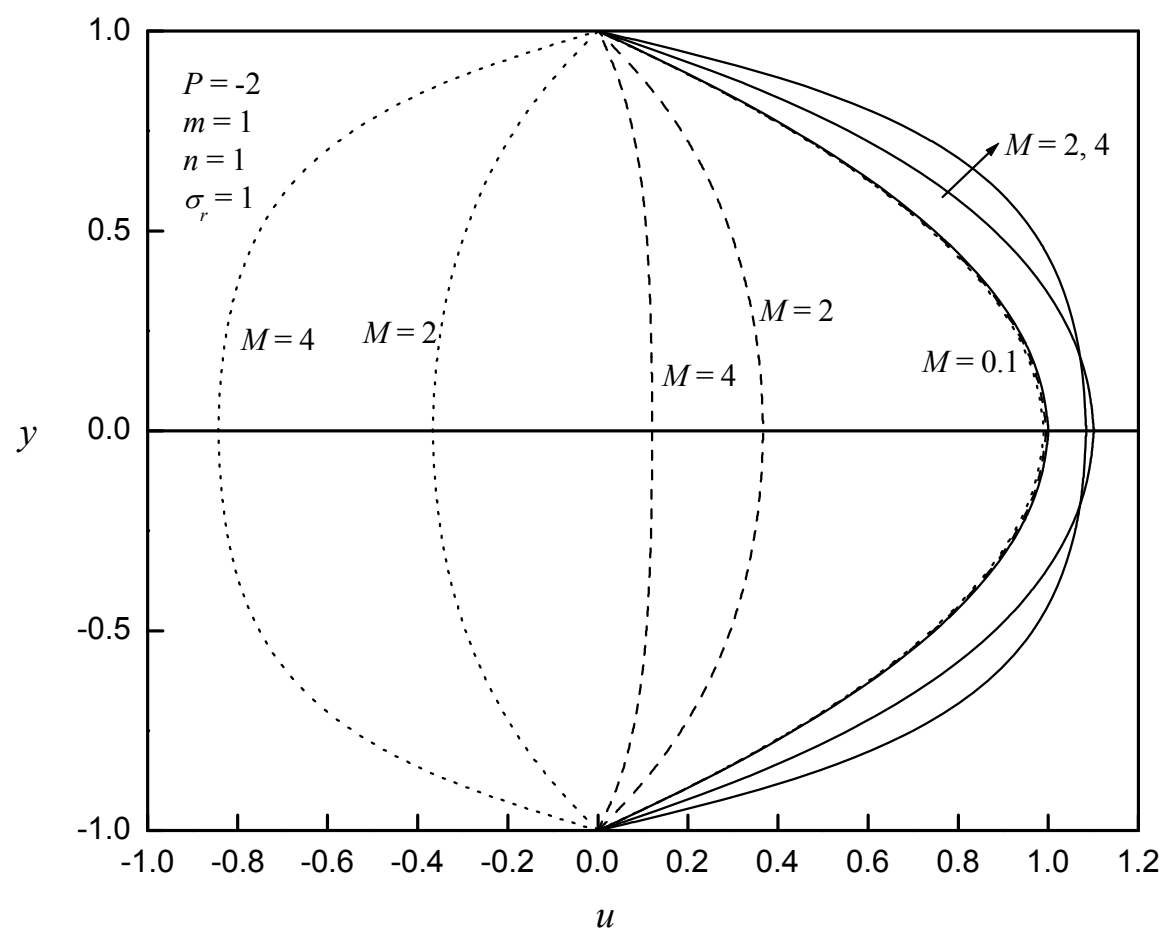

Fig.2. Velocity profiles for different values of the Hartman number M and electric load parameter $E$.

The effect of the Hartman number M, viscosity ratio $m$ and pressure gradient on ETDC for open and short circuits is shown in Tabs 1a, b, c. It is seen that the ETDC decreases with an increase in the Hartman number $\mathrm{M}$ for both open and short circuits. The results are compatible with the physics of the problem. In a Hartman flow, it is seen that the velocity profile becomes flatter with an increase of the Hartman number M. However, the ETDC decreases more rapidly with increasing the Hartman number M in a short circuited channel flow as compared with an open-circuited flow. 
As the viscosity ratio $m\left(\mu_{2} / \mu_{1}\right)$ increases, the ETDC $(F)$ decreases for values of $m<1$ and increases in magnitude for $m>1$ for both open and short circuits. This is due to the fact that viscosity ratio $m$ has a significant effect on velocity in region-2. The values of ETDC are symmetric for the pressure gradient $p>0$ and $p<0$. This is due to the fact that as $p$ increases for $p>0$, velocity increases which causes an increase in $F$ (it should be noted that $p$ is defined as $-\partial p / \partial x$ ).

Table 1a. Values of the effective dispersion coefficient for variations of the Hartman number, viscosity ratio, pressure gradients and electric load parameter in the absence of a first order chemical reaction.

\begin{tabular}{|l|l|l|l|}
\hline & \multicolumn{3}{|c|}{$E=-1$} \\
\hline $\mathrm{M}$ & $F_{1}\left(M, \sigma_{r}, m, p\right)$ & $F_{2}\left(M, \sigma_{r}, m, p\right)$ & $F\left(M, \sigma_{r}, m, p\right)$ \\
\hline 4 & 0.002260 & 0.002260 & 0.004521 \\
\hline 6 & 0.001823 & 0.001823 & 0.003646 \\
\hline 8 & 0.001341 & 0.001341 & 0.002681 \\
\hline 10 & $9.9643 E-4$ & $9.9643 E-4$ & 0.001993 \\
\hline 12 & $7.6092 E-4$ & $7.6092 E-4$ & 0.001522 \\
\hline$m$ & \multicolumn{3}{|l|}{} \\
\hline 0.1 & 2.349260 & 2.130990 & 4.480250 \\
\hline 0.5 & 0.025626 & 0.022898 & 0.048523 \\
\hline 1 & 0.001133 & 0.001133 & 0.002266 \\
\hline 2 & 0.008650 & 0.005368 & 0.014018 \\
\hline 3 & 0.012810 & 0.013202 & 0.026011 \\
\hline 4 & 0.009183 & 0.011565 & 0.020748 \\
\hline$p$ & \multicolumn{3}{|l}{} \\
\hline-15 & 0.045440 & 0.045440 & 0.090879 \\
\hline-10 & 0.024671 & 0.024671 & 0.049342 \\
\hline-5 & 0.010196 & 0.010196 & 0.020391 \\
\hline 0.1 & 0.001915 & 0.001915 & 0.003829 \\
\hline 5 & $1.2587 E-4$ & $1.2587 E-4$ & $2.5174 E-4$ \\
\hline 10 & 0.004531 & 0.004531 & 0.009063 \\
\hline 15 & 0.015230 & 0.015230 & 0.030461 \\
\hline 1 & 0.001133 & 0.001133 & 0.002266 \\
\hline$\sigma_{r}$ & & & 0.004446 \\
\hline 2 & 0.002939 & 0.001507 & 0.006807 \\
\hline 3 & 0.005228 & 0.001579 & 0.008847 \\
\hline 4 & 0.007163 & 0.001685 & 0.010551 \\
\hline 5 & 0.008730 & 0.001821 & \\
\hline
\end{tabular}


Table 1b. Values of the effective dispersion coefficient for variations of the Hartman number, viscosity ratio, pressure gradients and electric load parameter in the absence of a first order chemical reaction.

\begin{tabular}{|l|l|l|l|}
\hline & \multicolumn{3}{|c|}{$E=0$} \\
\hline $\mathrm{M}$ & $F_{1}\left(M, \sigma_{r}, m, p\right)$ & $F_{2}\left(M, \sigma_{r}, m, p\right)$ & $F\left(M, \sigma_{r}, m, p\right)$ \\
\hline 4 & $1.00464 E-5$ & $1.00464 E-5$ & $2.00927 E-5$ \\
\hline 6 & $1.48823 E-6$ & $1.48823 E-6$ & $2.97647 E-6$ \\
\hline 8 & $3.37759 E-7$ & $3.37759 E-7$ & $6.75518 E-6$ \\
\hline 10 & $1.01667 E-7$ & $1.01667 E-7$ & $2.03333 E-7$ \\
\hline 12 & $3.72109 E-8$ & $3.72109 E-8$ & $7.44218 E-8$ \\
\hline$m$ & \multicolumn{4}{|l|}{} \\
\hline 0.1 & $2.2388 E-5$ & 0.002 & 0.00202 \\
\hline 0.5 & $1.9476 E-4$ & $7.16 E-4$ & $9.1076 E-4$ \\
\hline 1 & $1.2587 E-4$ & $1.2587 E-4$ & $2.5174 E-4$ \\
\hline 2 & 0.0019 & $8.4955 E-4$ & 0.00275 \\
\hline 3 & 0.00108 & -0.00333 & -0.00224 \\
\hline 4 & -0.02861 & -0.02518 & -0.05378 \\
\hline$p$ & \multicolumn{3}{|l|}{} \\
\hline-15 & 0.0283211 & 0.0283211 & 0.0566422 \\
\hline-10 & 0.0125871 & 0.0125871 & 0.0251743 \\
\hline-5 & 0.0031468 & 0.0031468 & 0.0062936 \\
\hline 0.1 & $1.2587 E-6$ & $1.2587 E-6$ & $2.5174 E-6$ \\
\hline 5 & 0.0031468 & 0.0031468 & 0.0062936 \\
\hline 10 & 0.0125871 & 0.0125871 & 0.0251743 \\
\hline 15 & 0.0283211 & 0.0283211 & 0.0566422 \\
\hline$\sigma_{r}$ & \multicolumn{3}{|l}{} \\
\hline 1 & $5.0349 E-4$ & $5.0349 E-4$ & 0.001007 \\
\hline 2 & $7.8688 E-4$ & $6.1199 E-4$ & 0.001399 \\
\hline 3 & 0.001289 & $7.2795 E-4$ & 0.002017 \\
\hline 4 & 0.001725 & $7.7905 E-4$ & 0.002504 \\
\hline 5 & 0.002077 & $8.0366 E-4$ & 0.002880 \\
\hline
\end{tabular}
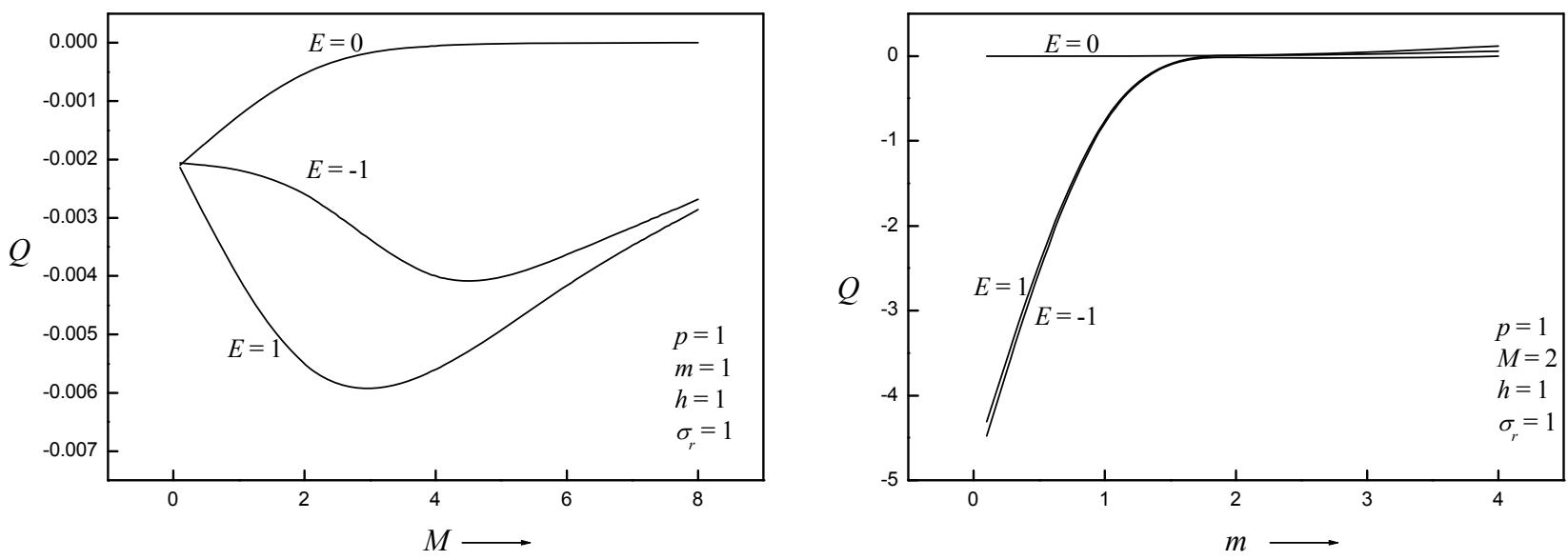

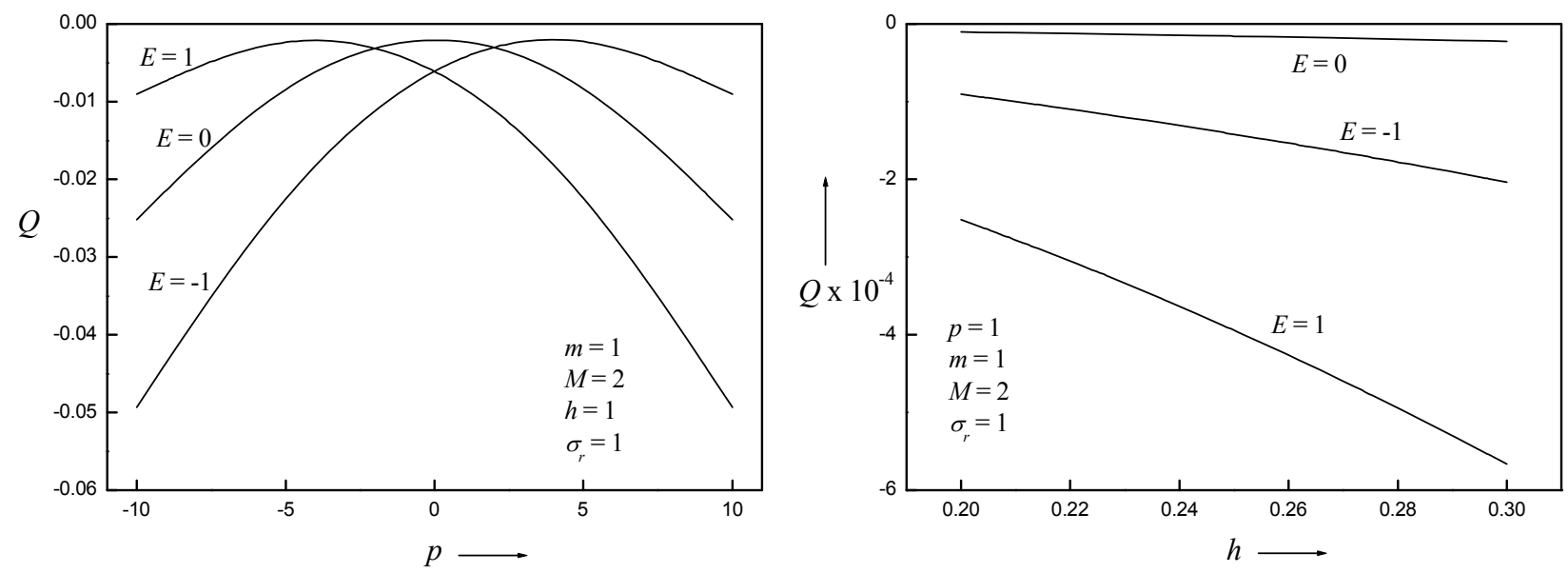

Fig.3. Volumetric flow rate $Q$ versus the Hartman number $M$, viscosity ratio $m$, pressure gradient $p$ height of the channel $h$ and electric load parameter in the absence of a first order chemical reaction.

Table 1c. Values of the effective dispersion coefficient for variations of the Hartman number, viscosity ratio, pressure gradients and electric load parameter in the absence of a first order chemical reaction.

\begin{tabular}{|l|l|l|l|}
\hline & \multicolumn{3}{|c|}{$E=1$} \\
\hline $\mathrm{M}$ & $F_{1}\left(M, \sigma_{r}, m, p\right)$ & $F_{2}\left(M, \sigma_{r}, m, p\right)$ & $F\left(M, \sigma_{r}, m, p\right)$ \\
\hline 4 & 0.002903 & 0.002903 & 0.005807 \\
\hline 6 & 0.002037 & 0.002037 & 0.004075 \\
\hline 8 & 0.001427 & 0.001427 & 0.002854 \\
\hline 10 & 0.001037 & 0.001037 & 0.002074 \\
\hline 12 & $7.82359 E-4$ & $7.82359 E-4$ & 0.001565 \\
\hline$m$ & \multicolumn{4}{|l|}{} \\
\hline 0.1 & 2.444240 & 1.868800 & 4.313040 \\
\hline 0.5 & 0.022215 & 0.017162 & 0.039377 \\
\hline 1 & 0.003147 & 0.003147 & 0.006294 \\
\hline 2 & 0.007024 & -0.011538 & -0.004513 \\
\hline 3 & 0.004653 & -0.046234 & -0.041582 \\
\hline 4 & -0.003975 & -0.111673 & -0.115648 \\
\hline$p$ & \multicolumn{4}{|l}{} \\
\hline-15 & 0.015230 & 0.015230 & 0.030461 \\
\hline-10 & 0.004531 & 0.004531 & 0.009063 \\
\hline-5 & $1.2587 E-4$ & $1.2587 E-4$ & $2.5174 E-4$ \\
\hline 0.1 & 0.002116 & 0.002116 & 0.004232 \\
\hline 5 & 0.010196 & 0.010196 & 0.020391 \\
\hline 10 & 0.024671 & 0.024671 & 0.049342 \\
\hline 15 & 0.045440 & 0.045440 & 0.090879 \\
\hline$\sigma_{r}$ & \multicolumn{5}{|l}{} \\
\hline 1 & 0.003147 & 0.003147 & 0.006294 \\
\hline 2 & 0.003528 & 0.002630 & 0.006158 \\
\hline 3 & 0.003972 & 0.002093 & 0.006065 \\
\hline 4 & 0.004334 & 0.001759 & 0.006093 \\
\hline 5 & 0.004619 & 0.001550 & 0.006170 \\
\hline & \multicolumn{5}{|l}{} \\
\hline
\end{tabular}


The effects of the Hartman number $\mathrm{M}$, viscosity ratio $m$, pressure gradient and height of the channel $h$ on the volumetric flow rate $Q$ is shown in Fig.2. As the Hartman number M increases the volumetric flow rate $Q$ decreases for $M=3$ for $E=-1$ and $E=0$ whereas it increases for $E=1$ and remains invariant for $M>3$ for both open and short circuits. The volumetric flow rate increases as the viscosity ratio $m$ increases up to $m=1.5$ (approximately) and remains constant for both open and short circuits. However the effect of $m$ on $Q$ is larger for an open circuit when compared to a short circuit. The Volumetric flow rate is symmetric for negative and positive values of the pressure gradient $p$ and the optimal flow rate is attained in the absence of the pressure gradient for both open and short circuits. The flow rate for $E=0$ lies between $E= \pm 1$ for $p<0$ and $p>0$. As the height of the channel $h$ increases, the volumetric flow rate decreases in magnitude for both open and short circuits. However the magnitude of retardation is larger for an open circuit when compared to a short circuit.

The results obtained (two-fluid model) in the absence of a chemical reactions agree with the results obtained by Gupta and Chatterjee [45] for the effect of the Hartman number M on the ETDC for a short circuit. That is, as $\mathrm{M}$ increases $F$ decreases. Letting $M \rightarrow 0$ and fixing $m=1, p=1$ and $h=1$ (i.e., considering the same fluid in both the regions) we obtain the results of Gupta and Gupta [37] for $K C=0$ which are also the results of Wooding [43] as shown in Tab.4.

\section{Case 2: Diffusion of a tracer with a combined homogeneous and heterogeneous first-order chemical reaction.}

The ETDC $F_{1}$ (region-1) and $F_{2}$ (region-2) for various values of the viscosity ratio $m$, pressure gradient $p$, and Hartman number $\mathrm{M}$ for a homogeneous chemical reaction for open and short circuit is shown in Tabs $2 \mathrm{a}, \mathrm{b}, \mathrm{c}$, respectively. As the reaction rate parameter $\alpha$ increases the total ETDC $\left(F=F_{1}+F_{2}\right)$ decreases for all values of $m, p$ and $M$ for both open and short circuits. This is due to the fact that an increase in $\alpha$ signifies the increasing number of moles of the solute undergoing a chemical reaction results in a drop in the dispersion coefficient. As the viscosity ratio $m$ increases, the ETDC decreases for values of $m \leq 1$ and increases for $m \geq 1$ for both open and short circuits. ETDC decreases as $p$ increases for $p<1$ and increases as $p$ increases for $p>1$ for $E=-1$ and 0 , whereas it increases in magnitude for both $p>1$ and $p<1$ for $E=1$.

As the Hartman number increases the ETDC decreases for $E=-1$ and 0 . For the electric field parameter $E=1$, the ETDC decreases as M increases for $M=0.1$ and $M=5$ whereas the ETDC increases in magnitude for values of $M>5$ as seen in Tab.2b. That is, as the Hartman number M increases, the ETDC, the reaction rate parameter $\alpha$ and the wall catalytic parameter $\beta$ decrease. Let $M \rightarrow 0, m=1, p=1$ then the present model agrees with the results of Gupta and Gupta [42] for both homogeneous and heterogeneous chemical reactions as shown in Tab.4. For a short circuit the effect of the Hartman number, reaction rate parameter $\alpha$ and wall catalytic parameter $\beta$, ETDC for the present model (two-fluid model) agree with the results of Sundhanshu et al. [44] (one fluid model). 
Table 2a. Values of the effective dispersion coefficient for variations of the reaction rate parameter $\alpha$, Hartman number $\mathrm{M}$, viscosity ratio $m$, pressure gradients $p$ and electric load parameter $E$ in the presence of a first order chemical reaction.

\begin{tabular}{|c|c|c|c|c|c|c|}
\hline \multicolumn{7}{|c|}{$E=-1$} \\
\hline & \multicolumn{3}{|c|}{$m=0.1$} & \multicolumn{3}{|c|}{$m=1$} \\
\hline$\alpha$ & $F_{1}\left(\alpha_{1}, \alpha_{2}\right)$ & $F_{2}\left(\alpha_{1}, \alpha_{2}\right)$ & $F\left(\alpha_{1}, \alpha_{2}\right)$ & $F_{1}\left(\alpha_{1}, \alpha_{2}\right)$ & $F_{2}\left(\alpha_{1}, \alpha_{2}\right)$ & $F\left(\alpha_{1}, \alpha_{2}\right)$ \\
\hline 0.4 & 2.761310 & 2.548470 & 5.309780 & 0.002226 & 0.002226 & 0.004452 \\
\hline 0.8 & 2.341920 & 2.173110 & 4.515030 & 0.002129 & 0.002129 & 0.004258 \\
\hline 1.2 & 1.871380 & 1.750980 & 3.622370 & 0.001985 & 0.001985 & 0.003971 \\
\hline 1.6 & 1.463460 & 1.383620 & 2.847080 & 0.001815 & 0.001815 & 0.003630 \\
\hline 2.0 & 1.145730 & 1.095930 & 2.241660 & 0.001636 & 0.001636 & 0.003272 \\
\hline$\alpha$ & \multicolumn{3}{|c|}{$m=2$} & \multicolumn{3}{|c|}{$p_{1}=p_{2}=-5$} \\
\hline 0.4 & 0.005810 & 0.007913 & 0.013723 & 0.004363 & 0.004363 & 0.008726 \\
\hline 0.8 & 0.005139 & 0.006757 & 0.011896 & 0.004173 & 0.004173 & 0.008346 \\
\hline 1.2 & 0.004362 & 0.005451 & 0.009813 & 0.003891 & 0.003891 & 0.007783 \\
\hline 1.6 & 0.003655 & 0.004308 & 0.007963 & 0.003557 & 0.003557 & 0.007115 \\
\hline 2.0 & 0.003069 & 0.003407 & 0.006476 & 0.003206 & 0.003206 & 0.006413 \\
\hline$\alpha$ & \multicolumn{3}{|c|}{$p_{1}=p_{2}=0.1$} & \multicolumn{3}{|c|}{$p_{1}=p_{2}=5$} \\
\hline 0.4 & 0.002501 & 0.002501 & 0.005002 & 0.001197 & 0.001197 & 0.002394 \\
\hline 0.8 & 0.002392 & 0.002392 & 0.004784 & 0.001145 & 0.001145 & 0.002290 \\
\hline 1.2 & 0.002231 & 0.002231 & 0.004462 & 0.001068 & 0.001068 & 0.002135 \\
\hline 1.6 & 0.002039 & 0.002039 & 0.004079 & $9.76079 E-4$ & $9.76079 E-4$ & 0.001952 \\
\hline 2.0 & 0.001838 & 0.001838 & 0.003676 & $8.79748 E-4$ & $8.79748 E-4$ & 0.001760 \\
\hline$\alpha$ & \multicolumn{3}{|c|}{$M=0.1$} & \multicolumn{3}{|c|}{$M=5$} \\
\hline 0.4 & 0.001012 & 0.001012 & 0.002024 & 0.002055 & 0.002055 & 0.004110 \\
\hline 0.8 & $9.66401 E-4$ & $9.66401 E-4$ & 0.001933 & 0.001967 & 0.001967 & 0.003934 \\
\hline 1.2 & $8.98947 E-4$ & $8.98947 E-4$ & 0.001798 & 0.001837 & 0.001837 & 0.003673 \\
\hline 1.6 & $8.19049 E-4$ & $8.19049 E-4$ & 0.001638 & 0.001682 & 0.001682 & 0.003364 \\
\hline 2.0 & $7.35214 E-4$ & $7.35214 E-4$ & 0.001470 & 0.001519 & 0.001519 & 0.003038 \\
\hline$\alpha$ & \multicolumn{3}{|c|}{$M=10$} & \multicolumn{3}{|c|}{$\sigma_{r}=0.1$} \\
\hline 0.4 & $9.82728 E-4$ & $9.82728 E-4$ & 0.001965 & 0.003985 & 0.007798 & 0.011783 \\
\hline 0.8 & $9.44031 E-4$ & $9.44031 E-4$ & 0.001888 & 0.003582 & 0.006766 & 0.010349 \\
\hline 1.2 & $8.86594 E-4$ & $8.86594 E-4$ & 0.001773 & 0.003104 & 0.005583 & 0.008687 \\
\hline 1.6 & $8.18166 E-4$ & $8.18166 E-4$ & 0.001636 & 0.002654 & 0.004523 & 0.007177 \\
\hline 2.0 & $7.45802 E-4$ & $7.45802 E-4$ & 0.001492 & 0.002267 & 0.003664 & 0.005931 \\
\hline$\alpha$ & \multicolumn{3}{|c|}{$\sigma_{r}=1$} & \multicolumn{3}{|c|}{$\sigma_{r}=2$} \\
\hline 0.4 & 0.002226 & 0.002226 & 0.004452 & 0.003218 & 0.001694 & 0.004911 \\
\hline 0.8 & 0.002129 & 0.002129 & 0.004258 & 0.002964 & 0.001652 & 0.004617 \\
\hline 1.2 & 0.001985 & 0.001985 & 0.003971 & 0.002646 & 0.001576 & 0.004222 \\
\hline 1.6 & 0.001815 & 0.001815 & 0.003630 & 0.002324 & 0.001470 & 0.003794 \\
\hline 2.0 & 0.001636 & 0.001636 & 0.003272 & 0.002028 & 0.001348 & 0.003376 \\
\hline
\end{tabular}


Table 2b. Values of the effective dispersion coefficient for variations of the reaction rate parameter $\alpha$, Hartman number $\mathrm{M}$, viscosity ratio $m$, pressure gradients $p$ and electric load parameter $E$ in the presence of a first order chemical reaction.

\begin{tabular}{|c|c|c|c|c|c|c|}
\hline & \multicolumn{6}{|c|}{$E=0$} \\
\hline & \multicolumn{3}{|c|}{$m=0.1$} & \multicolumn{3}{|c|}{$m=1$} \\
\hline$\alpha$ & $F_{1}\left(\alpha_{1}, \alpha_{2}\right)$ & $F_{2}\left(\alpha_{1}, \alpha_{2}\right)$ & $F\left(\alpha_{1}, \alpha_{2}\right)$ & $F_{1}\left(\alpha_{1}, \alpha_{2}\right)$ & $F_{2}\left(\alpha_{1}, \alpha_{2}\right)$ & $F\left(\alpha_{1}, \alpha_{2}\right)$ \\
\hline 0.4 & $8.00589 E-6$ & $1.49616 E-4$ & $1.57622 E-4$ & $9.8934 E-6$ & $9.8934 E-6$ & $1.97868 E-5$ \\
\hline 0.8 & $8.35876 E-6$ & $1.36619 E-4$ & $1.44978 E-4$ & $9.46219 E-6$ & $9.46219 E-6$ & $1.89244 E-5$ \\
\hline 1.2 & $8.50548 E-6$ & $1.20835 E-4$ & $1.29341 E-4$ & $8.82393 E-6$ & $8.82393 E-6$ & $1.76479 E-5$ \\
\hline 1.6 & $8.30537 E-6$ & $1.05557 E-4$ & $1.13862 E-4$ & $8.06677 E-6$ & $8.06677 E-6$ & $1.61335 E-5$ \\
\hline 2.0 & $7.82051 E-6$ & $9.20293 E-5$ & $9.98498 E-4$ & $7.27065 E-6$ & $7.27065 E-6$ & $1.45413 E-5$ \\
\hline$\alpha$ & \multicolumn{3}{|c|}{$m=2$} & \multicolumn{3}{|c|}{$p_{1}=p_{2}=-5$} \\
\hline 0.4 & $6.297 E-5$ & $2.11697 E-5$ & $8.41398 E-5$ & $2.47335 E-4$ & $2.47335 E-4$ & $4.9467 E-4$ \\
\hline 0.8 & $5.85902 E-5$ & $2.08786 E-5$ & $7.94688 E-5$ & $2.36555 E-4$ & $2.36555 E-4$ & $4.73109 E-4$ \\
\hline 1.2 & $5.29001 E-5$ & $2.01194 E-5$ & $7.30195 E-5$ & $2.20598 E-4$ & $2.20598 E-4$ & $4.41196 E-4$ \\
\hline 1.6 & $4.69458 E-5$ & $1.88913 E-5$ & $6.58371 E-5$ & $2.01669 E-4$ & $2.01669 E-4$ & $4.03338 E-4$ \\
\hline 2.0 & $4.12782 E-5$ & $1.73595 E-5$ & $5.86377 E-5$ & $1.81766 E-4$ & $1.81766 E-4$ & $3.63532 E-4$ \\
\hline$\alpha$ & \multicolumn{3}{|c|}{$p_{1}=p_{2}=0.1$} & \multicolumn{3}{|c|}{$p_{1}=p_{2}=5$} \\
\hline 0.4 & $9.8934 E-8$ & $9.8934 E-8$ & $1.97868 E-7$ & $2.47335 E-4$ & $2.47335 E-4$ & $4.9467 E-4$ \\
\hline 0.8 & $9.46219 E-8$ & $9.46219 E-8$ & $1.89244 E-7$ & $2.36555 E-4$ & $2.36555 E-4$ & $4.73109 E-4$ \\
\hline 1.2 & $8.82393 E-8$ & $8.82393 E-8$ & $1.76479 E-7$ & $2.20598 E-4$ & $2.20598 E-4$ & $4.41196 E-4$ \\
\hline 1.6 & $8.06677 E-8$ & $8.06677 E-8$ & $1.61335 E-7$ & $2.01669 E-4$ & $2.01669 E-4$ & $4.03338 E-4$ \\
\hline 2.0 & $7.27065 E-8$ & $7.27065 E-8$ & $1.45413 E-7$ & $1.81766 E-4$ & $1.81766 E-4$ & $3.63532 E-4$ \\
\hline$\alpha$ & \multicolumn{3}{|c|}{$M=0.1$} & \multicolumn{3}{|c|}{$M=5$} \\
\hline 0.4 & 0.00103000 & 0.00103000 & 0.00206514 & $3.56801 E-6$ & $3.56801 E-6$ & $7.13602 E-6$ \\
\hline 0.8 & $9.86023 E-4$ & $9.86023 E-4$ & 0.00197205 & $3.41511 E-6$ & $3.41511 E-6$ & $6.83022 E-6$ \\
\hline 1.2 & $9.17199 E-4$ & $9.17199 E-4$ & 0.00183440 & $3.18869 E-6$ & $3.18869 E-6$ & $6.37737 E-6$ \\
\hline 1.6 & $8.35679 E-4$ & $8.35679 E-4$ & 0.00167136 & $2.91988 E-6$ & $2.91988 E-6$ & $5.83976 E-6$ \\
\hline 2.0 & $7.50142 E-4$ & $7.50142 E-4$ & 0.00150028 & $2.63695 E-6$ & $2.63695 E-6$ & $5.27390 E-6$ \\
\hline$\alpha$ & \multicolumn{3}{|c|}{$M=10$} & \multicolumn{3}{|c|}{$\sigma_{r}=0.1$} \\
\hline 0.4 & $1.00268 \mathrm{E}-7$ & $1.00268 \mathrm{E}-7$ & $2.00536 E-7$ & $2.68126 E-4$ & $1.74734 E-4$ & $4.42859 E-4$ \\
\hline 0.8 & $9.63199 E-8$ & $9.63199 E-8$ & $1.92640 \mathrm{E}-7$ & $2.3091 E-4$ & $1.57484 E-4$ & $3.88394 E-4$ \\
\hline 1.2 & $9.04596 E-8$ & $9.04596 E-8$ & $1.80919 \mathrm{E}-7$ & $1.88526 E-4$ & $1.37013 E-4$ & $3.25539 E-4$ \\
\hline 1.6 & $8.34778 E-8$ & $8.34778 E-8$ & $1.66956 \mathrm{E}-7$ & $1.50963 E-4$ & $1.17758 E-4$ & $2.68721 E-4$ \\
\hline 2.0 & $7.60945 E-8$ & $7.60945 E-8$ & $1.52189 E-7$ & $1.20901 E-4$ & $1.01186 E-4$ & $2.22087 E-4$ \\
\hline$\alpha$ & \multicolumn{3}{|c|}{$\sigma_{r}=1$} & \multicolumn{3}{|c|}{$\sigma_{r}=2$} \\
\hline 0.4 & $9.8934 E-6$ & $9.8934 E-6$ & $1.97868 E-5$ & $1.04941 E-5$ & $1.63515 E-5$ & $2.68456 E-5$ \\
\hline 0.8 & $9.46219 E-6$ & $9.46219 E-6$ & $1.89244 E-5$ & $9.62562 E-5$ & $1.41923 E-5$ & $2.38179 E-5$ \\
\hline 1.2 & $8.82393 E-6$ & $8.82393 E-6$ & $1.76479 E-5$ & $8.56359 E-5$ & $1.17153 E-5$ & $2.02789 E-5$ \\
\hline 1.6 & $8.06677 E-6$ & $8.06677 E-6$ & $1.61335 E-5$ & $7.52492 E-5$ & $9.49710 E-5$ & $1.7022 E-5$ \\
\hline 2.0 & $7.27065 E-6$ & $7.27065 E-6$ & $1.45413 E-5$ & $6.59314 E-5$ & $7.69917 E-5$ & $1.42923 E-5$ \\
\hline
\end{tabular}


Table 2c. Values of the effective dispersion coefficient for variations of the reaction rate parameter $\alpha$, Hartman number $\mathrm{M}$, viscosity ratio $m$, pressure gradients $p$ and electric load parameter $E$ in the presence of a first order chemical reaction.

\begin{tabular}{|c|c|c|c|c|c|c|}
\hline & \multicolumn{6}{|c|}{$E=1$} \\
\hline & \multicolumn{3}{|c|}{$m=0.1$} & \multicolumn{3}{|c|}{$m=1$} \\
\hline$\alpha$ & $F_{1}\left(\alpha_{1}, \alpha_{2}\right)$ & $F_{2}\left(\alpha_{1}, \alpha_{2}\right)$ & $F\left(\alpha_{1}, \alpha_{2}\right)$ & $F_{1}\left(\alpha_{1}, \alpha_{2}\right)$ & $F_{2}\left(\alpha_{1}, \alpha_{2}\right)$ & $F\left(\alpha_{1}, \alpha_{2}\right)$ \\
\hline 0.4 & 2.748030 & 2.500990 & 5.249030 & 0.002859 & 0.002859 & 0.005718 \\
\hline 0.8 & 2.330730 & 2.133620 & 4.464350 & 0.002735 & 0.002735 & 0.005469 \\
\hline 1.2 & 1.862520 & 1.720360 & 3.582870 & 0.002550 & 0.002550 & 0.005100 \\
\hline 1.6 & 1.456600 & 1.360530 & 2.817130 & 0.002331 & 0.002331 & 0.004663 \\
\hline 2.0 & 1.140410 & 1.078570 & 2.218980 & 0.002101 & 0.002101 & 0.004202 \\
\hline$\alpha$ & \multicolumn{3}{|c|}{$m=2$} & \multicolumn{3}{|c|}{$p_{1}=p_{2}=-5$} \\
\hline 0.4 & 0.005893 & 0.008481 & 0.014374 & 0.001197 & 0.001197 & 0.002394 \\
\hline 0.8 & 0.005353 & 0.007296 & 0.012649 & 0.001145 & 0.001145 & 0.002290 \\
\hline 1.2 & 0.004703 & 0.005948 & 0.010651 & 0.001068 & 0.001068 & 0.002135 \\
\hline 1.6 & 0.004080 & 0.004756 & 0.008836 & $9.76079 E-4$ & $9.76079 E-4$ & 0.001952 \\
\hline 2.0 & 0.003532 & 0.003805 & 0.007338 & $8.79748 E-4$ & $8.79748 E-4$ & 0.001760 \\
\hline$\alpha$ & \multicolumn{3}{|c|}{$p_{1}=p_{2}=0.1$} & \multicolumn{3}{|c|}{$p_{1}=p_{2}=5$} \\
\hline 0.4 & 0.002564 & 0.002564 & 0.005129 & 0.004363 & 0.004363 & 0.008726 \\
\hline 0.8 & 0.002453 & 0.002453 & 0.004905 & 0.004173 & 0.004173 & 0.008346 \\
\hline 1.2 & 0.002287 & 0.002287 & 0.004574 & 0.003891 & 0.003891 & 0.007783 \\
\hline 1.6 & 0.002091 & 0.002091 & 0.004182 & 0.003557 & 0.003557 & 0.007115 \\
\hline 2.0 & 0.001885 & 0.001885 & 0.003769 & 0.003206 & 0.003206 & 0.006413 \\
\hline$\alpha$ & \multicolumn{3}{|c|}{$M=0.1$} & \multicolumn{3}{|c|}{$M=5$} \\
\hline 0.4 & 0.001053 & 0.001053 & 0.002107 & 0.002412 & 0.002412 & 0.004824 \\
\hline 0.8 & 0.001006 & 0.001006 & 0.002012 & 0.002309 & 0.002309 & 0.004617 \\
\hline 1.2 & $9.35635 E-4$ & $9.35635 E-4$ & 0.001871 & 0.002156 & 0.002156 & 0.004311 \\
\hline 1.6 & $8.52476 E-4$ & $8.52476 E-4$ & 0.001705 & 0.001974 & 0.001974 & 0.003948 \\
\hline 2.0 & $7.6522 E-4$ & $7.6522 E-4$ & 0.001530 & 0.001783 & 0.001783 & 0.003565 \\
\hline$\alpha$ & \multicolumn{3}{|c|}{$M=10$} & \multicolumn{3}{|c|}{$\sigma_{r}=0.1$} \\
\hline 0.4 & 0.001023 & 0.001023 & 0.002046 & 0.002770 & 0.006362 & 0.009132 \\
\hline 0.8 & $9.82559 E-4$ & $9.82559 E-4$ & 0.001965 & 0.002664 & 0.005725 & 0.008389 \\
\hline 1.2 & $9.22778 E-4$ & $9.22778 E-4$ & 0.001846 & 0.002501 & 0.004959 & 0.007460 \\
\hline 1.6 & $8.51557 E-4$ & $8.51557 E-4$ & 0.001703 & 0.002301 & 0.004229 & 0.006529 \\
\hline 2.0 & $7.7624 E-4$ & $7.7624 E-4$ & 0.001552 & 0.002085 & 0.003592 & 0.005677 \\
\hline$\alpha$ & \multicolumn{3}{|c|}{$\sigma_{r}=1$} & \multicolumn{3}{|c|}{$\sigma_{r}=2$} \\
\hline 0.4 & 0.002859 & 0.002859 & 0.005718 & 0.003355 & 0.001978 & 0.005333 \\
\hline 0.8 & 0.002735 & 0.002735 & 0.005469 & 0.003135 & 0.001928 & 0.005063 \\
\hline 1.2 & 0.002550 & 0.002550 & 0.005100 & 0.002847 & 0.001836 & 0.004683 \\
\hline 1.6 & 0.002331 & 0.002331 & 0.004663 & 0.002543 & 0.001710 & 0.004253 \\
\hline 2.0 & 0.002101 & 0.002101 & 0.004202 & 0.002250 & 0.001565 & 0.003815 \\
\hline
\end{tabular}

Tables 3a, b, c display the variations of the ETDC on the wall catalytic parameter $\beta$, viscosity ratio $m$, pressure gradient $p$ and Hartman number $M$ for a fixed value of the homogeneous reaction rate parameter $\alpha\left(=\alpha_{1}=\alpha_{2}\right)$ for both open and short circuits, respectively. The effects of $m, p$ and the 
Hartman number M on the ETDC show the same results as observed for a homogeneous chemical reaction for both open and short circuits (Tables $2 a, b, c)$.

Table 3a. Values of the effective dispersion coefficient for variations of wall catalytic parameter $\beta$, Hartman number $\mathrm{M}$, viscosity ratio $m$, and pressure gradients $p$ in the presence of a first order chemical reaction.

\begin{tabular}{|c|c|c|c|c|c|c|}
\hline & \multicolumn{6}{|c|}{$E=-1$} \\
\hline & \multicolumn{3}{|c|}{$m=0.1$} & \multicolumn{3}{|c|}{$m=1$} \\
\hline$\beta$ & $F_{l}\left(\alpha_{i}, \beta_{i}\right)$ & $F_{2}\left(\alpha_{i}, \beta_{i}\right)$ & $F\left(\alpha_{i}, \beta_{i}\right)$ & $F_{l}\left(\alpha_{i}, \beta_{i}\right)$ & $F_{2}\left(\alpha_{i}, \beta_{i}\right)$ & $F\left(\alpha_{i}, \beta_{i}\right)$ \\
\hline 2 & 1.984610 & 1.898800 & 3.883410 & 0.002018 & 0.002018 & 0.004037 \\
\hline 4 & 1.402270 & 1.566430 & 2.968710 & 0.001850 & 0.001850 & 0.003699 \\
\hline 6 & 1.136290 & 1.399450 & 2.535740 & 0.001791 & 0.001791 & 0.003582 \\
\hline 8 & 0.995491 & 1.308140 & 2.303630 & 0.001764 & 0.001764 & 0.003528 \\
\hline 10 & 0.908249 & 1.250680 & 2.158930 & 0.001748 & 0.001748 & 0.003496 \\
\hline$\beta$ & \multicolumn{3}{|c|}{$m=2$} & \multicolumn{3}{|c|}{$p_{1}=p_{2}=-5$} \\
\hline 2 & 0.004817 & 0.005622 & 0.010439 & 0.003956 & 0.003956 & 0.007912 \\
\hline 4 & 0.004966 & 0.003443 & 0.008409 & 0.003625 & 0.003625 & 0.007251 \\
\hline 6 & 0.004956 & 0.002509 & 0.007465 & 0.003511 & 0.003511 & 0.007022 \\
\hline 8 & 0.004936 & 0.002027 & 0.006963 & 0.003457 & 0.003457 & 0.006914 \\
\hline 10 & 0.004919 & 0.001731 & 0.006650 & 0.003426 & 0.003426 & 0.006852 \\
\hline$\beta$ & \multicolumn{3}{|c|}{$p_{1}=p_{2}=0.1$} & \multicolumn{3}{|c|}{$p_{1}=p_{2}=5$} \\
\hline 2 & 0.002268 & 0.002268 & 0.004536 & 0.001085 & 0.001085 & 0.002171 \\
\hline 4 & 0.002078 & 0.002078 & 0.004157 & $9.94742 E-4$ & $9.94742 E-4$ & 0.001989 \\
\hline 6 & 0.002013 & 0.002013 & 0.004025 & $9.63291 E-4$ & $9.63291 E-4$ & 0.001927 \\
\hline 8 & 0.001982 & 0.001982 & 0.003964 & $9.48563 E-4$ & $9.48563 E-4$ & 0.001897 \\
\hline 10 & 0.001964 & 0.001964 & 0.003928 & $9.40021 E-4$ & $9.40021 E-4$ & 0.001880 \\
\hline$\beta$ & \multicolumn{3}{|c|}{$M=0.1$} & \multicolumn{3}{|c|}{$M=5$} \\
\hline 2 & $9.17127 E-4$ & $9.17127 E-4$ & 0.001834 & 0.001865 & 0.001865 & 0.003729 \\
\hline 4 & $8.48274 E-4$ & $8.48274 E-4$ & 0.001697 & 0.001704 & 0.001704 & 0.003407 \\
\hline 6 & $8.24406 E-4$ & $8.24406 E-4$ & 0.001649 & 0.001648 & 0.001648 & 0.003296 \\
\hline 8 & $8.13229 E-4$ & $8.13229 E-4$ & 0.001626 & 0.001622 & 0.001622 & 0.003243 \\
\hline 10 & $8.06746 E-4$ & $8.06746 E-4$ & 0.001613 & 0.001607 & 0.001607 & 0.003213 \\
\hline$\beta$ & \multicolumn{3}{|c|}{$M=10$} & \multicolumn{3}{|c|}{$\sigma_{r}=0.1$} \\
\hline 0.4 & $8.95191 E-4$ & $8.95191 E-4$ & 0.001790 & 0.003447 & 0.005719 & 0.009166 \\
\hline 0.8 & $8.09645 E-4$ & $8.09645 E-4$ & 0.001619 & 0.003797 & 0.003661 & 0.007458 \\
\hline 1.2 & $7.7999 E-4$ & $7.7999 E-4$ & 0.001560 & 0.003892 & 0.002786 & 0.006679 \\
\hline 1.6 & $7.66102 E-4$ & $7.66102 E-4$ & 0.001532 & 0.003930 & 0.002336 & 0.006266 \\
\hline 2.0 & $7.58048 E-4$ & $7.58048 E-4$ & 0.001516 & 0.003950 & 0.002061 & 0.006011 \\
\hline$\beta$ & \multicolumn{3}{|c|}{$\sigma_{r}=1$} & \multicolumn{3}{|c|}{$\sigma_{r}=2$} \\
\hline 0.4 & 0.002018 & 0.002018 & 0.004037 & 0.002658 & 0.001657 & 0.004315 \\
\hline 0.8 & 0.001850 & 0.001850 & 0.003699 & 0.002011 & 0.001835 & 0.003846 \\
\hline 1.2 & 0.001791 & 0.001791 & 0.003582 & 0.001754 & 0.001911 & 0.003665 \\
\hline 1.6 & 0.001764 & 0.001764 & 0.003528 & 0.001625 & 0.001951 & 0.003576 \\
\hline 2.0 & 0.001748 & 0.001748 & 0.003496 & 0.001547 & 0.001975 & 0.003522 \\
\hline
\end{tabular}


Table 3b. Values of the effective dispersion coefficient for variations of the wall catalytic parameter $\beta$, Hartman number $\mathrm{M}$, viscosity ratio $m$, and pressure gradients $p$ in the presence of a first order chemical reaction.

\begin{tabular}{|c|c|c|c|c|c|c|}
\hline & \multicolumn{6}{|c|}{$E=0$} \\
\hline & \multicolumn{3}{|c|}{$m=0.1$} & \multicolumn{3}{|c|}{$m=1$} \\
\hline$\beta$ & $F_{l}\left(\alpha_{i}, \beta_{i}\right)$ & $F_{2}\left(\alpha_{i}, \beta_{i}\right)$ & $F\left(\alpha_{i}, \beta_{i}\right)$ & $F_{l}\left(\alpha_{i}, \beta_{i}\right)$ & $F_{2}\left(\alpha_{i}, \beta_{i}\right)$ & $F\left(\alpha_{i}, \beta_{i}\right)$ \\
\hline 2 & $8.80015 E-6$ & $1.25461 E-4$ & $1.34261 E-4$ & $8.97079 E-6$ & 8.97079 E-6 & $1.79416 E-5$ \\
\hline 4 & $1.06137 E-5$ & $1.09507 E-4$ & $1.20121 E-4$ & 8.22100 E-6 & $8.22100 E-6$ & $1.6442 E-5$ \\
\hline 6 & $1.15313 E-5$ & $1.0242 E-4$ & $1.13951 E-4$ & $7.96109 E-6$ & $7.96109 E-6$ & $1.59222 E-5$ \\
\hline 8 & $1.20341 E-5$ & $9.8707 E-5$ & $1.10741 E-4$ & $7.83936 E-6$ & $7.83936 E-6$ & $1.56787 E-5$ \\
\hline 10 & $1.23509 E-5$ & $9.64181 E-5$ & $1.08769 E-4$ & $7.76877 E-6$ & $7.76877 E-6$ & $1.55375 E-5$ \\
\hline$\beta$ & \multicolumn{3}{|c|}{$m=2$} & \multicolumn{3}{|c|}{$p_{1}=p_{2}=-5$} \\
\hline 2 & $5.42686 E-5$ & $2.04981 E-5$ & $7.47668 E-5$ & $2.2427 E-5$ & $2.2427 E-5$ & $4.48539 E-5$ \\
\hline 4 & $4.7363 E-5$ & $2.06885 E-5$ & $6.80515 E-5$ & $2.05525 E-5$ & $2.05525 E-5$ & $4.1105 E-5$ \\
\hline 6 & $4.4527 E-5$ & $2.09914 E-5$ & $6.55185 E-5$ & $1.99027 E-5$ & $1.99027 E-5$ & $3.98054 E-5$ \\
\hline 8 & $4.3087 E-5$ & $2.11933 E-5$ & $6.42803 E-5$ & $1.95984 E-5$ & $1.95984 E-5$ & $3.91968 E-5$ \\
\hline 10 & $4.22133 E-5$ & $2.1331 E-5$ & $6.35443 E-5$ & $1.94219 E-5$ & $1.94219 E-5$ & $3.88438 E-5$ \\
\hline$\beta$ & \multicolumn{3}{|c|}{$p_{1}=p_{2}=0.1$} & \multicolumn{3}{|c|}{$p_{1}=p_{2}=5$} \\
\hline 2 & $8.97079 E-8$ & $8.97079 E-8$ & $1.79416 E-7$ & $2.2427 E-5$ & $2.2427 E-5$ & $4.48539 E-5$ \\
\hline 4 & $8.22100 E-8$ & $8.22100 E-8$ & $1.64420 \mathrm{E}-7$ & $2.05525 E-5$ & $2.05525 E-5$ & $4.1105 E-5$ \\
\hline 6 & $7.96109 E-8$ & $7.96109 E-8$ & $1.59222 E-7$ & $1.99027 E-5$ & $1.99027 E-5$ & $3.98054 E-5$ \\
\hline 8 & $7.83936 E-8$ & $7.83936 E-8$ & $1.56787 E-7$ & $1.95984 E-5$ & $1.95984 E-5$ & $3.91968 E-5$ \\
\hline 10 & $7.76877 E-8$ & $7.76877 E-8$ & $1.55375 E-7$ & $1.94219 E-5$ & $1.94219 E-5$ & $3.884388-5$ \\
\hline$\beta$ & \multicolumn{3}{|c|}{$M=0.1$} & \multicolumn{3}{|c|}{$M=5$} \\
\hline 2 & $9.35748 E-4$ & $9.35748 E-4$ & 0.00187150 & $3.23731 E-6$ & $3.23731 E-6$ & $6.47463 E-6$ \\
\hline 4 & $8.65498 E-4$ & $8.65498 E-4$ & 0.00173100 & $2.95774 E-6$ & $2.95774 E-6$ & $5.91549 E-6$ \\
\hline 6 & $8.41145 E-4$ & $8.41145 E-4$ & 0.00168229 & $2.86083 E-6$ & $2.86083 E-6$ & $5.72166 E-6$ \\
\hline 8 & $8.2974 E-4$ & $8.2974 E-4$ & 0.00165948 & $2.81544 E-6$ & $2.81544 E-6$ & 5.63089 E-6 \\
\hline 10 & $8.23126 E-4$ & $8.23126 E-4$ & 0.00164625 & $2.78912 E-6$ & $2.78912 E-6$ & $5.57824 E-6$ \\
\hline$\beta$ & \multicolumn{3}{|c|}{$M=10$} & \multicolumn{3}{|c|}{$\sigma_{r}=0.1$} \\
\hline 0.4 & $9.13367 E-8$ & $9.13367 E-8$ & $1.82673 E-7$ & $1.9335 E-4$ & $1.51182 E-4$ & $3.44532 E-4$ \\
\hline 0.8 & $8.26084 E-8$ & $8.26084 E-8$ & $1.65217 \mathrm{E}-7$ & $1.19893 E-4$ & $1.64294 E-4$ & $2.84187 E-4$ \\
\hline 1.2 & $7.95827 E-8$ & $7.95827 E-8$ & $1.59165 \mathrm{E}-7$ & $8.88153 E-5$ & $1.67752 E-4$ & $2.56567 E-4$ \\
\hline 1.6 & $7.81657 E-8$ & $7.81657 E-8$ & $1.56331 \mathrm{E}-7$ & $7.28403 E-5$ & $1.69096 E-4$ & $2.41936 E-4$ \\
\hline 2.0 & $7.73439 E-8$ & $7.73439 E-8$ & $1.54688 E-7$ & $6.30865 E-5$ & $1.6978 E-4$ & $2.32867 E-4$ \\
\hline$\beta$ & \multicolumn{3}{|c|}{$\sigma_{r}=1$} & \multicolumn{3}{|c|}{$\sigma_{r}=2$} \\
\hline 0.4 & 8.97079 E-6 & $8.97079 E-6$ & $1.79416 E-5$ & $9.42998 E-6$ & $1.188666-5$ & $2.13166 E-5$ \\
\hline 0.8 & 8.22100 E-6 & $8.22100 E-6$ & $1.6442 E-5$ & $1.06612 E-5$ & 7.14330 E-6 & $1.78044 E-5$ \\
\hline 1.2 & $7.96109 E-6$ & 7.96109E-6 & $1.59222 E-5$ & $1.10628 E-5$ & $5.16619 E-6$ & $1.6229 E-5$ \\
\hline 1.6 & 7.83936 E-6 & $7.83936 E-6$ & $1.56787 E-5$ & $1.12445 E-5$ & $4.15603 E-6$ & $1.54005 E-5$ \\
\hline 2.0 & $7.76877 E-6$ & $7.76877 E-6$ & $1.55375 E-5$ & $1.13477 E-5$ & $3.54119 E-6$ & $1.48889 E-5$ \\
\hline
\end{tabular}


Table 3c. Values of the effective dispersion coefficient for variations of the wall catalytic parameter $\beta$, Hartman number $\mathrm{M}$, viscosity ratio $m$, and pressure gradients $p$ in the presence of a first order chemical reaction.

\begin{tabular}{|c|c|c|c|c|c|c|}
\hline & \multicolumn{6}{|c|}{$E=1$} \\
\hline & \multicolumn{3}{|c|}{$m=0.1$} & \multicolumn{3}{|c|}{$m=1$} \\
\hline$\beta$ & $F_{1}\left(\alpha_{i}, \beta_{i}\right)$ & $F_{2}\left(\alpha_{i}, \beta_{i}\right)$ & $F\left(\alpha_{i}, \beta_{i}\right)$ & $F_{l}\left(\alpha_{i}, \beta_{i}\right)$ & $F_{2}\left(\alpha_{i}, \beta_{i}\right)$ & $F\left(\alpha_{i}, \beta_{i}\right)$ \\
\hline 2 & 1.973670 & 1.866970 & 3.840630 & 0.002593 & 0.002593 & 0.005185 \\
\hline 4 & 1.388180 & 1.549230 & 2.937410 & 0.002376 & 0.002376 & 0.004752 \\
\hline 6 & 1.121350 & 1.388600 & 2.509950 & 0.002301 & 0.002301 & 0.004602 \\
\hline 8 & 0.980221 & 1.300590 & 2.280810 & 0.002266 & 0.002266 & 0.004531 \\
\hline 10 & 0.892810 & 1.245150 & 2.137960 & 0.002245 & 0.002245 & 0.004490 \\
\hline$\beta$ & \multicolumn{3}{|c|}{$m=2$} & \multicolumn{3}{|c|}{$p_{1}=p_{2}=-5$} \\
\hline 2 & 0.005195 & 0.006053 & 0.011248 & 0.001085 & 0.001085 & 0.002171 \\
\hline 4 & 0.005781 & 0.003521 & 0.009302 & $9.94742 E-4$ & $9.94742 E-4$ & 0.001989 \\
\hline 6 & 0.005954 & 0.002460 & 0.008414 & $9.63291 E-4$ & $9.63291 E-4$ & 0.001927 \\
\hline 8 & 0.006028 & 0.001917 & 0.007945 & $9.48563 E-4$ & $9.48563 E-4$ & 0.001897 \\
\hline 10 & 0.006068 & 0.001586 & 0.007655 & $9.40021 E-4$ & $9.40021 E-4$ & 0.001880 \\
\hline$\beta$ & \multicolumn{3}{|c|}{$p_{1}=p_{2}=0.1$} & \multicolumn{3}{|c|}{$p_{1}=p_{2}=5$} \\
\hline 2 & 0.002325 & 0.002325 & 0.004651 & 0.003956 & 0.003956 & 0.007912 \\
\hline 4 & 0.002131 & 0.002131 & 0.004262 & 0.003625 & 0.003625 & 0.007251 \\
\hline 6 & 0.002064 & 0.002064 & 0.004127 & 0.003511 & 0.003511 & 0.007022 \\
\hline 8 & 0.002032 & 0.002032 & 0.004064 & 0.003457 & 0.003457 & 0.006914 \\
\hline 10 & 0.002014 & 0.002014 & 0.004027 & 0.003426 & 0.003426 & 0.006852 \\
\hline$\beta$ & \multicolumn{3}{|c|}{$M=0.1$} & \multicolumn{3}{|c|}{$M=5$} \\
\hline 2 & $9.54557 E-4$ & $9.54557 E-4$ & 0.001909 & 0.002188 & 0.002188 & 0.004377 \\
\hline 4 & $8.82894 E-4$ & $8.82894 E-4$ & 0.001766 & 0.001999 & 0.001999 & 0.003999 \\
\hline 6 & $8.58052 E-4$ & $8.58052 E-4$ & 0.001716 & 0.001934 & 0.001934 & 0.003868 \\
\hline 8 & $8.46418 E-4$ & $8.46418 E-4$ & 0.001693 & 0.001903 & 0.001903 & 0.003806 \\
\hline 10 & $8.39671 E-4$ & $8.39671 E-4$ & 0.001679 & 0.001885 & 0.001885 & 0.003771 \\
\hline$\beta$ & \multicolumn{3}{|c|}{$M=10$} & \multicolumn{3}{|c|}{$\sigma_{r}=0.1$} \\
\hline 0.4 & $9.31725 E-4$ & $9.31725 E-4$ & 0.001863 & 0.002702 & 0.005009 & 0.007710 \\
\hline 0.8 & $8.42688 E-4$ & $8.42688 E-4$ & 0.001685 & 0.003180 & 0.003526 & 0.006706 \\
\hline 1.2 & $8.11823 E-4$ & $8.11823 E-4$ & 0.001624 & 0.003366 & 0.002919 & 0.006285 \\
\hline 1.6 & $7.97369 E-4$ & $7.97369 E-4$ & 0.001595 & 0.003459 & 0.002611 & 0.006070 \\
\hline 2.0 & $7.88985 E-4$ & $7.88985 E-4$ & 0.001578 & 0.003514 & 0.002424 & 0.005938 \\
\hline$\beta$ & \multicolumn{3}{|c|}{$\sigma_{r}=1$} & \multicolumn{3}{|c|}{$\sigma_{r}=2$} \\
\hline 0.4 & 0.002593 & 0.002593 & 0.005185 & 0.002868 & 0.001900 & 0.004769 \\
\hline 0.8 & 0.002376 & 0.002376 & 0.004752 & 0.002328 & 0.001979 & 0.004307 \\
\hline 1.2 & 0.002301 & 0.002301 & 0.004602 & 0.002117 & 0.002020 & 0.004137 \\
\hline 1.6 & 0.002266 & 0.002266 & 0.004531 & 0.002012 & 0.002043 & 0.004055 \\
\hline 2.0 & 0.002245 & 0.002245 & 0.004490 & 0.001949 & 0.002057 & 0.004007 \\
\hline
\end{tabular}


Table 4. Values of the effective dispersion coefficient.

\begin{tabular}{|l|l|c|c|c|}
\hline & \multicolumn{3}{|c|}{ Two fluid model (present model) } & $\begin{array}{c}\text { One fluid model } \\
\text { Gupta and Gupta [42] }\end{array}$ \\
\hline & \multicolumn{4}{|c|}{ In the absence of first-order chemical reaction } \\
\hline$m=p$ & $F_{1}(m, p)$ & $F_{2}(m, p)$ & $F(m, p)$ & $F(m, p)$ \\
\hline 1 & 0.0010582 & 0.0010582 & 0.0021164 & 0.0021164 \\
\hline \multicolumn{4}{|c|}{ In the presence of first-order homogeneous chemical reaction } \\
\hline$\alpha$ & $F_{1}\left(\alpha_{1}, \alpha_{2}\right)$ & $F_{2}\left(\alpha_{1}, \alpha_{2}\right)$ & $F\left(\alpha_{1}, \alpha_{2}\right)$ & $F(\alpha)$ \\
\hline 0.4 & 0.0010099 & 0.0010099 & 0.0020199 & 0.00201987 \\
\hline 0.8 & $9.1846 E-4$ & $9.1846 E-4$ & 0.0018369 & 0.00183692 \\
\hline 1.2 & $8.2952 E-4$ & $8.2952 E-4$ & 0.0016590 & 0.00165904 \\
\hline 1.6 & $7.474 E-4$ & $7.474 E-4$ & 0.0014948 & 0.00149480 \\
\hline 2 & $6.70579 E-4$ & $6.70579 E-4$ & 0.0013412 & 0.00134116 \\
\hline \multicolumn{5}{|c|}{ In the presence of first-order combined homogeneous and heterogeneous } \\
\hline$\beta$ & $F_{1}\left(\alpha_{i}, \beta_{i}\right)$ & $F_{2}\left(\alpha_{i}, \beta_{i}\right)$ & $F\left(\alpha_{i}, \beta_{i}\right)$ & $F(\alpha, \beta)$ \\
\hline 2 & $8.48463 E-4$ & $8.48463 E-4$ & 0.00169693 & 0.0016969 \\
\hline 4 & $8.30289 E-4$ & $8.30289 E-4$ & 0.00166058 & 0.0016606 \\
\hline 6 & $8.22866 E-4$ & $8.22866 E-4$ & 0.00164573 & 0.0016457 \\
\hline 8 & $8.18832 E-4$ & $8.18832 E-4$ & 0.00163766 & 0.0016377 \\
\hline 10 & $8.16298 E-4$ & $8.16298 E-4$ & 0.00163260 & 0.0016326 \\
\hline
\end{tabular}

\section{Conclusion}

1. The ETDC decreases with an increase in the Hartman number for $E=-1$ and $E=0$ whereas it decreases for $M<1$ and increases for $M>1$ for $E=1$ with or without chemical reactions.

2. The ETDC decreases for $m \leq 1$ and $p<1$ whereas it increases for $m>1$ and $p>1$ as $m$ and $p$ increases for both open and short circuits in the absence or in the presence of chemical reactions.

3. As the homogeneous reaction rate parameter and wall catalytic parameter increases, the ETDC decreases for all values of the viscosity ratio, pressure gradient and Hartman number for both open and short circuits.

4. The results for the two fluid model (present model) agree with the results for one fluid models of Gupta and Chatterjee [45], Gupta and Gupta [42] and Wooding [43].

\section{Acknowledgements}

One of the authors JCU thanks UGC-New Delhi for the financial support under UGC-Major Research Project.

\section{Appendix}

Case 1: Diffusion of a tracer in the absence of a first-order chemical reaction.

$$
A=M^{2} E+\frac{d p_{1}^{*}}{d x}, \quad B=\frac{\sigma_{r} M^{2}}{m}, \quad c=\frac{\sigma_{r} M^{2} E}{m^{2} n}+\frac{d p_{2}^{*}}{d x}, \quad a_{4}=\frac{c-M^{2} a_{3} \cosh (B)}{M^{2} \sinh (B)}
$$




$$
\begin{aligned}
& a_{3}=\frac{-M\left(\left(\frac{A}{M^{2}}-\frac{m n c}{B^{2}}\right) \cosh (M) \sinh (B)-\frac{A}{M^{2}} \sinh (B)\right)}{M m n \cosh (M) \sinh (B)+m^{2} n B \cosh (B) \sinh (M)}, \quad a_{1}=\frac{A}{M^{2}}+m n a_{3}-\frac{m n c}{B^{2}}, \\
& a_{2}=\frac{M^{2} a_{1} \cosh (M)-A}{M^{2} \sinh (M)}, \quad b_{11}=\frac{a_{1} \sinh (M)}{M}-\frac{a_{2} \cosh (M)}{M}+l_{1}, \\
& b_{32}=-\frac{a_{3} \sinh (B)}{B}-\frac{a_{4} \cosh (B)}{B}-l_{2} .
\end{aligned}
$$

Case 1b: Diffusion of a tracer in the absence of a first order chemical reaction and for a purely viscous fluid (two fluid model)

$$
p_{1}=\frac{d p_{1}^{*}}{d x}, \quad p_{2}=\frac{d p_{2}^{*}}{d x}, \quad a_{2}=m n a_{4}, \quad a_{1}=m^{2} n a_{3}, \quad a_{4}=-\frac{p_{2}}{2}-a_{3}, \quad a_{3}=\frac{p_{1}-p_{2} m n}{2(m+1) m n}
$$

Case 2a: Diffusion of a tracer in the presence of a homogeneous first-order chemical reaction.

$$
\begin{aligned}
& g_{1}=\frac{-a_{1} M \sinh (M)}{M^{2}-\alpha_{1}^{2}}+\frac{a_{2} M \cosh (M)}{M^{2}-\alpha_{1}^{2}}, \quad g_{2}=\frac{a_{3} B \sinh (B)}{B^{2}-\alpha_{2}^{2}}+\frac{a_{4} B \cosh (B)}{B^{2}-\alpha_{2}^{2}}, \\
& g_{3}=\frac{a_{1}}{M^{2}-\alpha_{1}^{2}}-\frac{l_{1}}{\alpha_{1}^{2}}, \quad g_{4}=-\frac{a_{3}}{B^{2}-\alpha_{2}^{2}}+\frac{l_{2}}{\alpha_{2}^{2}}, \quad g_{5}=\frac{a_{2} M}{M^{2}-\alpha_{1}^{2}}, \quad g_{3}=-\frac{D a_{4} B}{B^{2}-\alpha_{2}^{2}}, \\
& D r=D \alpha_{2}^{2} \cosh \left(\alpha_{1}\right) \sinh \left(\alpha_{2}\right)+\alpha_{1} \alpha_{2} \sinh \left(\alpha_{1}\right) \cosh \left(\alpha_{2}\right), \\
& b_{41}=\frac{-1}{D r}\left(g_{3} \alpha_{1} \alpha_{2} \sinh \left(\alpha_{1}\right) \sinh \left(\alpha_{2}\right)-g_{5} \alpha_{2} \cosh \left(\alpha_{1}\right) \sinh \left(\alpha_{2}\right)+g_{1} \alpha_{2} \sinh \left(\alpha_{2}\right)\right), \\
& b_{42}=\frac{-1}{D r}\left(g_{4} \alpha_{1} \alpha_{2} \sinh \left(\alpha_{1}\right) \sinh \left(\alpha_{2}\right)-g_{6} \alpha_{2} \cosh \left(\alpha_{1}\right) \sinh \left(\alpha_{2}\right)+g_{2} \alpha_{1} \sinh \left(\alpha_{1}\right)\right), \\
& b_{31}=-\frac{b_{41} \cosh \left(\alpha_{2}\right)}{\sinh \left(\alpha_{2}\right)}, \quad b_{32}=\frac{-b_{42} \alpha_{2} \cosh \left(\alpha_{2}\right)+g_{2}}{\alpha_{2} \sinh \left(\alpha_{2}\right)}, \quad b_{11}=b_{31}-g_{3}, \quad b_{12}=b_{32}-g_{4}, \\
& b_{21}=\frac{b_{11} \alpha_{1} \sinh \left(\alpha_{1}\right)-g_{1}}{\alpha_{1} \cosh \left(\alpha_{1}\right)}, \quad b_{22}=\frac{b_{12} \sinh \left(\alpha_{1}\right)}{\cosh \left(\alpha_{1}\right)}, \\
& C_{11}=-b_{11} \cosh \left(\alpha_{1} \eta\right)-b_{21} \sinh \left(\alpha_{1} \eta\right)-\frac{a_{1}}{M^{2}} \cosh (M \eta)-\frac{a_{2}}{M^{2}} \sinh (M \eta)-\frac{l_{1}}{2} \eta^{2}, \\
& C_{11}=-b_{12} \cosh \left(\alpha_{1} \eta\right)-b_{22} \sinh \left(\alpha_{1} \eta\right), \quad C_{21}=-b_{31} \cosh \left(\alpha_{2} \eta\right)-b_{41} \sinh \left(\alpha_{2} \eta\right),
\end{aligned}
$$




$$
C_{22}=b_{32} \cosh \left(\alpha_{2} \eta\right)+b_{42} \sinh \left(\alpha_{2} \eta\right)+\left(\frac{a_{3}}{B^{2}} \cosh (B \eta)+\frac{a_{4}}{B^{2}} \sinh (B \eta)+\frac{l_{2}}{2} \eta^{2}\right)
$$

Case 2b: Diffusion of a tracer with a combined homogeneous and heterogeneous first-order chemical reaction.

$$
\begin{aligned}
& g_{4}=-\alpha_{1} \sinh \left(\alpha_{1}\right)-\beta_{1} \cosh \left(\alpha_{1}\right), \quad g_{5}=\alpha_{1} \cosh \left(\alpha_{1}\right)+\beta_{1} \sinh \left(\alpha_{1}\right), \\
& g_{6}=-\frac{a_{1} M \sinh (M)}{M^{2}-\alpha_{1}^{2}}+\frac{a_{2} M \cosh (M)}{M^{2}-\alpha_{1}^{2}}-\beta_{1}\left(\frac{a_{1} \cosh (M)}{M^{2}-\alpha_{1}^{2}}-\frac{a_{2} \sinh (M)}{M^{2}-\alpha_{1}^{2}}-\frac{l_{1}}{\alpha_{1}^{2}}\right), \\
& g_{7}=\alpha_{2} \sinh \left(\alpha_{2}\right)+\beta_{2} \cosh \left(\alpha_{2}\right), \quad g_{8}=\alpha_{2} \cosh \left(\alpha_{2}\right)+\beta_{2} \sinh \left(\alpha_{2}\right), \\
& g_{9}=\frac{a_{3} B \sinh (B)}{B^{2}-\alpha_{2}^{2}}+\frac{a_{4} B \cosh (B)}{B^{2}-\alpha_{2}^{2}}+\beta_{2}\left(\frac{a_{3} \cosh (B)}{B^{2}-\alpha_{2}^{2}}+\frac{a_{4} \sinh (B)}{B^{2}-\alpha_{2}^{2}}-\frac{l_{2}}{\alpha_{2}^{2}}\right), \\
& b_{41}=\frac{\alpha_{1}}{g_{5} g_{6} D \alpha_{2}-g_{4} g_{5} \alpha_{1}}\left(g_{4} g_{7}\left(\frac{a_{1}}{M^{2}-\alpha_{1}^{2}}-\frac{l_{1}}{\alpha_{1}^{2}}\right)-\frac{g_{5} g_{7} M a_{2}}{\alpha_{1}\left(M^{2}-\alpha_{1}^{2}\right)}-g_{6} g_{7}\right), \\
& b_{42}=\frac{\alpha_{1}}{g_{5} g_{6} D \alpha_{2}-g_{4} g_{5} \alpha_{1}}\left(-g_{4} g_{7} l_{3}-\frac{g_{5} g_{7} D l_{2}}{\alpha_{1}}+g_{4} g_{9}\right)-g_{4} g_{6}\left(\frac{a_{3}}{B^{2}-\alpha_{2}^{2}}-\frac{l_{2}}{\alpha_{2}^{2}}\right), \\
& b_{31}=-\frac{g_{8} b_{41}}{g_{7}}, \quad b_{32}=\frac{-g_{8} b_{42}-g_{9}}{g_{7}}, \quad b_{11}=b_{31}-\frac{a_{1}}{M^{2}-\alpha_{1}^{2}}+\frac{l_{1}}{\alpha_{1}^{2}}, \\
& b_{12}=b_{32}+l_{5}, \quad b_{21}=\frac{-g_{4} b_{11}-g_{6}}{g_{5}}, \quad b_{22}=-\frac{g_{4} b_{12}}{g_{5}} .
\end{aligned}
$$

Case 2c: Diffusion of a tracer in the presence of a homogeneous first-order chemical reaction in the absence of the magnetic field for a purely viscous fluid (two fluid model).

$$
\begin{aligned}
& l c_{1}=-\frac{p_{1}}{12}+\frac{a_{1}}{4}+\frac{a_{2}}{2}-\frac{p_{2}}{12}-\frac{a_{3}}{4}-\frac{a_{4}}{2}, \quad l_{2}=-\frac{p_{1}}{12}+\frac{a_{1}}{4}-\frac{a_{2}}{2}-\frac{p_{2}}{12}-\frac{a_{3}}{4}+\frac{a_{4}}{2}, \quad l_{1}=\frac{-p_{1}}{2 \alpha_{1}^{2}}, \\
& l_{2}=\frac{-a_{1}}{\alpha_{1}^{2}}, \quad l_{3}=-\frac{l c_{1}}{\alpha_{1}^{2}}-\frac{p_{1}}{\alpha_{1}^{4}}, \quad l_{4}=\frac{-p_{2}}{2 \alpha_{2}^{2}}, \quad l_{5}=\frac{-a_{3}}{\alpha_{2}^{2}}, \quad l_{6}=-\frac{l c_{2}}{\alpha_{2}^{2}}-\frac{p_{2}}{\alpha_{2}^{4}}, \\
& \operatorname{Dr}=\alpha_{1} \alpha_{2} \sinh \left(\alpha_{1}\right) \cosh \left(\alpha_{2}\right)+\alpha_{2}^{2} D \sinh \left(\alpha_{2}\right) \cosh \left(\alpha_{1}\right), \\
& b_{41}=\frac{-1}{\operatorname{Dr}}\left(l_{3} \alpha_{1} \alpha_{2} \sinh \left(\alpha_{1}\right) \sinh \left(\alpha_{2}\right)-l_{2} \alpha_{2} \cosh \left(\alpha_{1}\right) \sinh \left(\alpha_{2}\right)+\alpha_{2} \sinh \left(\alpha_{2}\right)\left(l_{2}-2 l_{1}\right)\right), \\
& b_{42}=\frac{-1}{\operatorname{Dr}}\left(\alpha_{1} \sinh \left(\alpha_{2}\right)\left(l_{5}+2 l_{4}\right)-l_{6} \alpha_{1} \alpha_{2} \sinh \left(\alpha_{1}\right) \sinh \left(\alpha_{2}\right)+l_{5} D \alpha_{2} \cosh \left(\alpha_{1}\right) \sinh \left(\alpha_{2}\right)\right),
\end{aligned}
$$




$$
\begin{aligned}
& b_{31}=\frac{-b_{41} \cosh \left(\alpha_{2}\right)}{\sinh \left(\alpha_{2}\right)}, \quad b_{32}=\frac{-b_{42} \alpha_{2} \cosh \left(\alpha_{2}\right)-2 l_{4}-l_{5}}{\alpha_{2} \sinh \left(\alpha_{2}\right)}, \quad b_{11}=b_{31}-l_{3}, \quad b_{12}=b_{32}+l_{6}, \\
& b_{21}=\frac{b_{11} \alpha_{1} \sinh \left(\alpha_{1}\right)+2 l_{1}-l_{2}}{\alpha_{1} \cosh \left(\alpha_{1}\right)}, \quad b_{22}=\frac{b_{12} \sinh \left(\alpha_{1}\right)}{\cosh \left(\alpha_{1}\right)} .
\end{aligned}
$$

\section{Nomenclature}

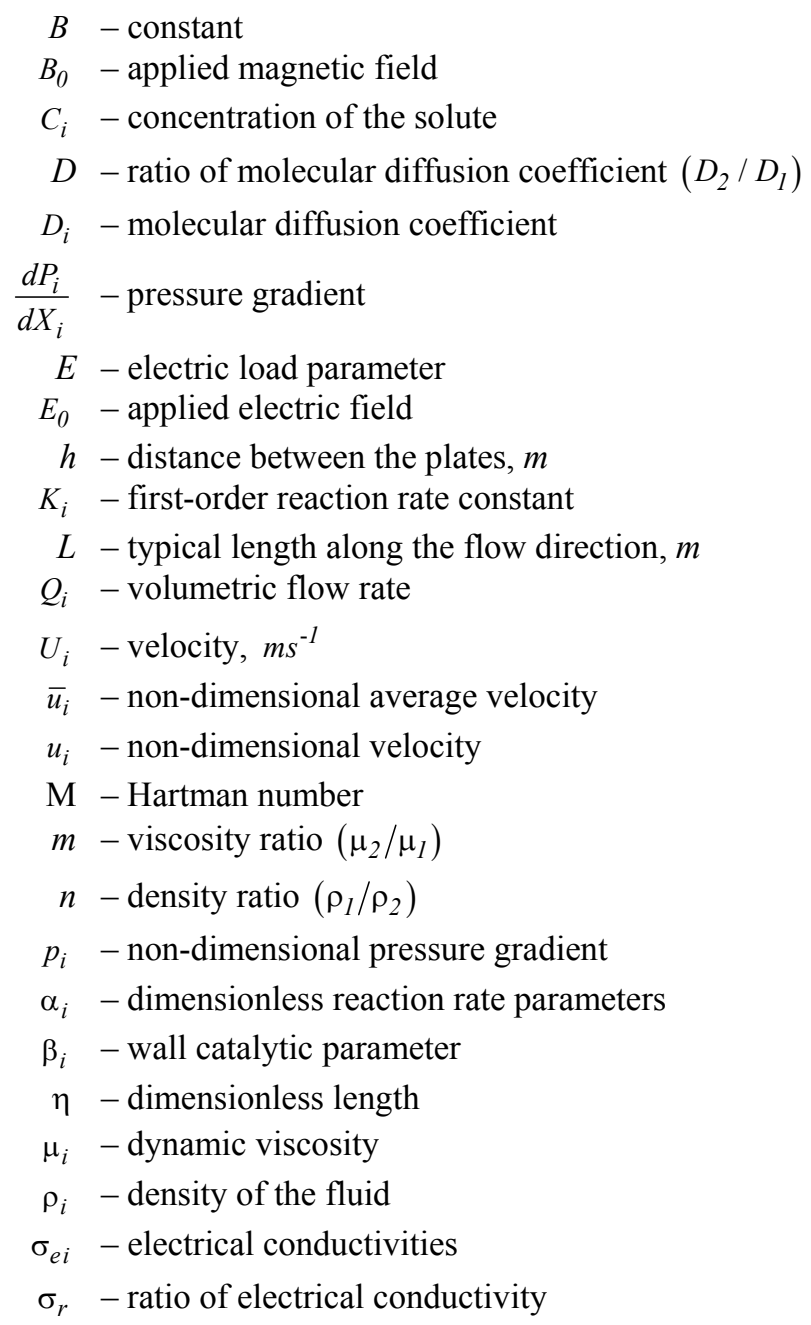

\section{Subscripts}

$i=1,2 \quad$ - where 1,2 -quantities for region- 1 and region-2, respectively

\section{References}

[1] Levenspiel O. and Smith W.K. (1957): Notes on the diffusion-type model for the longitudinal mixing of fluids in flow. - Chem. Engng. Sci., vol.6, pp.27-233.

[2] Danckwerts P.V. (1953): The effect of incomplete mixing on homogeneous reactions. - Chem. Engng. Sci., vol.8, No.1-2, pp.93-102. 
[3] Taylor G.I. (1953): Dispersion of soluble matter in solvent flowing slowly through a tube. - Proceedings of the Royal Society of London A, vol.219, pp.186-203

[4] Taylor G.I. (1954): The dispersion of matter in turbulent flow through a pipe. - Proceedings of the Royal Society of London A, 223, pp.446-468

[5] Taylor G.I. (1954): Conditions under which dispersion of a solute in a stream of solvent can be used to measure molecular diffusion. - Proceedings of the Royal Society of London A, 225, pp.473-477.

[6] Batchelor G.K. (1981): Preoccupations of a journal editor. - J. Fluid Mech., vol.106, pp.1-25.

[7] Aris R. (1956): On the dispersion of a solute in a fluid flowing through a tube. - Proceedings of Royal Society London A 235, pp.67-77.

[8] Horn F.J.M. and Kipp JR R.L. (1971): Induced transport in pulsating flow. - AIChE Journal, vol.17, pp.621-626.

[9] Brenner H. (1980): A general theory of Taylor dispersion phenomena. - Physicochem. Hydrodyn., vol.1, pp.91123.

[10] Brenner H. and Edwards D.A. (1982): Macrotransport Process. - Butterworth-Heinemann, Boston, 714.

[11] Philip J.R. (1963): The theory of dispersal during laminar flow in tubes. - I. Australian J. Physics, vol.16, pp.287299.

[12] Gill W.N. and Sankarasubramanian R. (1970): A note on the solution of transient dispersion problems.Proceedings of the Royal Society A, vol.316, pp.341-350.

[13] Gill W.N. and Sankarasubramanian R. (1972): Dispersion of non-uniformly distributed time-variable continuous sources in time-dependent flow. - Proceedings of Royal Society London A, vol.327, pp.191-208.

[14] DeGance A.E. and Johns L.E. (1978a): The theory of dispersion of chemically active solutes in a rectilinear flow field. - Appl. Sci. Res., vol.34, pp.189-225.

[15] DeGance A.E. and Johns L.E. (1980): On the construction of dispersion approximations to the solution of the convective diffusion equation. - AIChE Journal, vol.26, pp.411-419.

[16] Hatton T.A. and Lightfoot E.N. (1982): On the significance of the dispersion coefficient in two-phase flow. Chem. Engng. Sci., vol.37, pp.1289-1307.

[17] Hatton T.A. and Lightfoot E.N. (1984a): Dispersion, mass transfer and chemical reaction in multiphase contactors: part I: theoretical developments. - AIChE journal 30, pp.235-243.

[18] Hatton T.A. and Lightfoot E.N. (1984b): Dispersion, mass transfer and chemical reaction in multiphase contactors: Part II: Numerical examples. - AIChE Journal, vol.30, pp.243- 249.

[19] Yamanaka T. (1983): Projection operator theoretical approach to unsteady convective diffusion phenomena. J. Chem. Engng. Japan, vol.16, pp.29-35.

[20] Yamanaka T. (1983b): Generalization of Taylor's approximate solution for dispersion phenomena. - J. Chem. Engng. Japan, vol.16, pp.511-512.

[21] Yamanaka T. and Inui S. (1994): Taylor dispersion models involving nonlinear irreversible reactions. - J. Chem. Engng. Japan, vol.27, pp.434-435.

[22] Smith R. (1981): A delay-diffusion description for contaminant dispersion. - J. Fluid Mech., vol.105, pp.469-486.

[23] Smith R. (1987): Diffusion in shear flows made easy: the Taylor limit. - J. Fluid Mech., vol.175, pp.201-214.

[24] Cleland F.A. and Wilhelm R.H. (1956): Diffusion and reaction in viscous-flow tubular reactor. - AIChE Journal, vol.2, pp.489-497.

[25] Katz S. (1959): Chemical reactions catalysed on a tube wall. - Chem. Engng. Sci., vol.10, pp.202-211.

[26] Walker R. (1961): Chemical reaction and diffusion in a catalytic tubular reactor. - Physics of Fluids, vol.4, pp.1211-1216.

[27] Solomon R.L. and Hudson J.L. (1967): Heterogeneous and homogeneous reactions in a tubular reactor. - AIChE. J., vol.13, pp.545-550. 
[28] Packham B.A. and Shail R. (1971): Stratified laminar flow of two immiscible fluids. - Mathematical Proceedings Cambridge Philosophical Society, vol.69, pp.443-448.

[29] Alireza S. and Sahai V. (1990): Heat transfer in developing magnetohydrodynamic Poiseuille flow and variable transport properties. - Int. J. Heat and Mass Transfer, vol.33, pp.1711-1720.

[30] Malashetty M.S. and Leela V. (1991): Magnetohydrodynamic heat transfer in two fluid flow. - Proc. of National Heat Transfer Conferences sponsored by AIChE and ASME- HTD, Phase Change Heat Transfer, vol.159, pp.171175.

[31] Malashetty M.S. and Leela V. (1992): Magnetohydrodynamic heat transfer in two-phase flow. - Int. J. Engng. Sci., vol.30, pp.371-377.

[32] Lohrasbi J. and Sahai V. (1988): Magnetohydrodynamic heat transfer in two-phase flow between parallel plates. Appl. Sci. Res., vol.45, pp.53-66.

[33] Malashetty M.S. and Umavathi J.C. (1997): Magnetohydrodynamic two phase flow in an inclined channel. - Int. J. Multiphase Flow, vol.23, pp.545-560.

[34] Chamkha A.J. (1999): Flow of two-immiscible fluids in porous and nonporous channels. - ASME. J. Fluids Eng., vol.122, pp.117-124.

[35] Malashetty M.S. Umavathi J.C. and Kumar J.P. (2001): Two fluid magneto convection flow in an inclined channel. - Int. J. Transport Phenomena, vol.3, pp.73-84.

[36] Malashetty M.S. Umavathi J.C. and Kumar J.P. (2001): Convective magneto hydrodynamic two fluid flow and heat transfer in an inclined channel. - Heat and Mass Transfer J., vol.37, pp.259-264.

[37] Malashetty M.S. Umavathi J.C. and Kumar J.P. (2001): Convective flow and heat transfer in an inclined composite porous medium. - J. Porous Media, vol.4, pp.15-22.

[38] Umavathi J.C., Liu I.C. and Kumar J.P. (2010): Magnetohydrodynamic Poseuille-Coutte flow and heat transfer in an inclined channel. - J. Mech., vol.26, pp.525-532.

[39] Umavathi J.C. and Shekar M. (2011): Mixed convective flow of two immiscible viscous fluids in a vertical wavy channel with traveling thermal waves. - Heat Transfer-Asian Res., vol.40, pp.721-743.

[40] Kumar J.P., Umavathi J.C. and Shivakumar M. (2011): Effect of first order chemical reaction on magneto convection of immiscible fluids in a vertical channel. - Heat Transfer Asian Res., vol.40, pp.608-640.

[41] Kumar J.P., Umavathi J.C., Chamkha A.J and Ashok Basawaraj (2012): Solute dispersion between two parallel plates containing porous and fluid layers. - J. Porous Media, vol.15, pp.1031-1047.

[42] Gupta A.S. and Chatterjee A.S. (1968): Dispersion of soluble matter in the hydromagnetic laminar flow between two parallel plates. - Mathematical Proceedings of the Cambridge Philosophical Society, vol.64, pp.1209-1214.

[43] Wooding R.A. (1960): Instability of a viscous liquid of variable density in a vertical Hele-Shaw cell. - J. Fluid Mech., vol.7, pp.501-515.

[44] Sudhanshu, Ghoshal K., Subhash Sikdar Ch. and Ajit K. (1976): Dispersion of solutes in laminar hydromagnetic flows with homogeneous and heterogeneous chemical reactions. - Proceedings of the Indian National Science Academy. Part A, Physical Sci., vol.43, pp.370-379.

[45] Gupta A.S. and Chatterjee A.S. (1968): Dispersion of soluble matter in the hydromagnetic laminar flow between two parallel plates. - In Mathematical Proceedings of the Cambridge Philosophical Society, vol.64, pp.1209-1214.

Received: October 16, 2015

Revised: June 5, 2016 